\title{
Non-Abelian Proca-Dirac-Higgs theory: Particlelike solutions and their energy spectrum
}

\author{
Vladimir Dzhunushaliev, ${ }^{1,2,3,4, *}$ Vladimir Folomeev, ${ }^{2,3, \dagger}$ and Arislan Makhmudov ${ }^{4, \$}$ \\ ${ }^{1}$ Department of Theoretical and Nuclear Physics, Al-Farabi Kazakh National University, \\ Almaty 050040, Kazakhstan \\ ${ }^{2}$ Institute of Experimental and Theoretical Physics, Al-Farabi Kazakh National University, \\ Almaty 050040, Kazakhstan \\ ${ }^{3}$ Institute of Physicotechnical Problems and Material Science of the NAS of the Kyrgyz Republic, \\ 265 a, Chui Street, Bishkek 720071, Kyrgyzstan \\ ${ }^{4}$ Institute of Systems Science, Durban University of Technology, 4000 Durban, South Africa
}

(Received 3 March 2019; published 16 April 2019)

\begin{abstract}
We study a system consisting of a non-Abelian SU(2) Proca field interacting with nonlinear scalar (Higgs) and spinor fields. For such a system, it is shown that particlelike solutions with finite energy do exist. It is demonstrated that the solutions depend on three free parameters of the system, including the central value of the scalar field $\xi_{0}$. For some fixed values of $\xi_{0}$, we find energy spectra of the solutions. It is shown that for each of the cases under consideration, there is a minimum value of the energy $\Delta=\Delta\left(\xi_{0}\right)$ [the mass gap $\Delta\left(\xi_{0}\right)$ for a fixed value of $\xi_{0}$ ]. The behavior of the function $\Delta\left(\xi_{0}\right)$ is studied for some range of $\xi_{0}$.
\end{abstract}

DOI: 10.1103/PhysRevD.99.076009

\section{INTRODUCTION}

By Proca theories one means gauge theories (both Abelian and non-Abelian ones) where the gauge invariance is violated explicitly by introducing a mass term. The first use of a Proca field was by Yukawa to describe pions. Later on the Proca theory has found applications in various fields of modern theoretical physics. The use of a Proca field results in the following consequences: a photon may acquire a rest mass, Einstein-Proca gravity involves a graviton of nonzero rest mass, Einstein-Proca-Weyl theories can be applied to describe dark matter [1], and using a real Proca field with a negative mass square, one can describe tachyons-particles moving with the velocity greater than the velocity of light [2].

Additionally, the following research involving a Proca field may be noted. Reference [3] studies Einstein-nonAbelian-Proca theory in an asymptotically anti-de Sitter spacetime with gauge group $\mathrm{SU}(2)$. The results obtained describe a gravitating Proca monopole. References [4] and [5] consider stars supported by Proca fields. In Ref. [6],

\footnotetext{
*v.dzhunushaliev@gmail.com

†vfolomeev@mail.ru

*arslan.biz@gmail.com
}

Published by the American Physical Society under the terms of the Creative Commons Attribution 4.0 International license. Further distribution of this work must maintain attribution to the author(s) and the published article's title, journal citation, and DOI. Funded by SCOAP. theories of the generalized Proca field are under investigation: the generalized Proca action for a vector field with derivative self-interactions with only three propagating degrees of freedom is constructed. The paper [7] also deals with the generalized Proca action for an Abelian vector field. The work [8] considers relativistic quantum mechanics of a Proca particle in Riemannian spacetimes. In Ref. [9] the authors have placed observational constraints on a class of dark energy models within the framework of generalized Proca theories. The purpose of the paper [10] is to build the first-order terms of the generalized SU(2) Proca theory and to discuss a general form of the complete theory.

In the present paper we study a non-Abelian SU(2) Proca field interacting with nonlinear scalar and spinor fields. The scalar field is described by the Klein-Gordon equation with the Higgs potential. The spinor field $\psi$ is described by the Dirac equation with a potential term of the form $|\bar{\psi} \psi|^{2}$. Our purpose here is to obtain particlelike spherically symmetric solutions and to study their energy spectra. We will show below that the energy spectrum depends on the parameters $f_{2}, E$, and $\phi_{0}$. The parameter $f_{2}$ describes a behavior of the $\mathrm{SU}(2)$ Proca field at the center of the system; $E / \hbar$ is a frequency of the stationary spinor field entering the factor $e^{-i E t / \hbar}$; the parameter $\phi_{0}$ is a central value of the Higgs field $\phi$. We show that the energy spectrum has a minimum, at least for some values of $\phi_{0}$, and we argue that this will also take place for any value of $\phi_{0}$ lying in the range $0<\phi_{0}<\infty$. The behavior of this minimum as $\phi_{0} \rightarrow \infty$ is of great interest: if in this limit the minimum is nonzero, 
one can say that there is a mass gap $\Delta \neq 0$ in non-Abelian Proca-Dirac-Higgs theory.

If such a mass gap does exist, this would be of great significance. The reason is that in quantum field theory there is a problem to prove the existence of a mass gap in quantum chromodynamics. Since this problem is highly nontrivial, one of the possible ways for solving it could be a consideration of simpler problems where quantum systems are replaced by approximate classical systems. In this case, if one could show that in such classical systems a mass gap can occur, this could be regarded as an indication of the possibility of existence of the mass gap in quantum systems. From this point of view, the classical system studied in the present paper and regarded as some approximation to realistic quantum systems can be of some interest.

Thus, the purpose of the present paper is to (i) obtain particlelike solutions within a theory with a non-Abelian SU(2) Proca field plus a Higgs scalar field plus a nonlinear Dirac field, (ii) study energy spectra of these solutions, (iii) search for a minimum of the spectrum (a mass gap), and (iv) understand the mechanism of the occurrence of a mass gap within the theory under investigation and, on this basis, to suggest a similar mechanism for QCD.

The paper is organized as follows. In Sec. II, we give the field equations describing a system consisting of a nonAbelian SU(2) Proca field interacting with nonlinear scalar and spinor fields. In Sec. III, we choose the Ansätze (stationary one for the spinor field and static ones for the Proca and scalar fields) solve to the equations of Sec. II, using which the corresponding complete set of equations is written down. For this set, in Sec. IV we numerically solve the equations and find regular solutions describing particlelike systems in non-Abelian Proca-Dirac-Higgs theory. In Sec. V, we construct the energy spectrum of the solutions obtained and show the existence of a mass gap for this spectrum. Finally, in Sec. VII, we summarize and discuss the results obtained.

\section{NON-ABELIAN PROCA PLUS HIGGS SCALAR AND NONLINEAR DIRAC FIELDS THEORY}

The Lagrangian describing a system consisting of a nonAbelian SU(2) Proca field $A_{\mu}^{a}$ interacting with nonlinear scalar, $\phi$, and spinor, $\psi$, fields can be taken in the form

$$
\begin{aligned}
\mathcal{L}= & -\frac{1}{4} F_{\mu \nu}^{a} F^{a \mu \nu}-\frac{\mu^{2}}{2} A_{\mu}^{a} A^{a \mu}+\frac{1}{2} \partial_{\mu} \phi \partial^{\mu} \phi+\frac{\lambda_{1}}{2} \phi^{2} A_{\mu}^{a} A^{a \mu} \\
& -\frac{\lambda_{2}}{4}\left(\phi^{2}-M^{2}\right)^{2}+i \hbar c \bar{\psi} \gamma^{\mu}\left(\partial_{\mu}-i \frac{g}{2} \sigma^{a} A_{\mu}^{a}\right) \psi \\
& +\frac{\Lambda}{2} g \hbar c \phi(\bar{\psi} \psi)^{2}-m_{f} c^{2} \bar{\psi} \psi
\end{aligned}
$$

Here $m_{f}$ is the mass of the spinor field; $D_{\mu}=\partial_{\mu}-i \frac{g}{2} \sigma^{a} A_{\mu}^{a}$ is the gauge derivative, where $g$ is the coupling constant and $\sigma^{a}$ are the $\mathrm{SU}(2)$ generators (the Pauli matrices); $F_{\mu \nu}^{a}=$ $\partial_{\mu} A_{\nu}^{a}-\partial_{\nu} A_{\mu}^{a}+g \epsilon_{a b c} A_{\mu}^{b} A_{\nu}^{c}$ is the tensor of the Proca field in non-Abelian SU(2) Proca theory, where $\epsilon_{a b c}$ (the completely antisymmetric Levi-Civita symbol) are the SU(2) structure constants; $\mu, M, \Lambda$, and $\lambda_{1,2}$ are constants; and $\gamma^{\mu}$ are the Dirac matrices in the standard representation.

The corresponding field equations are as follows:

$$
\begin{gathered}
D_{\nu} F^{a \mu \nu}-\left(\lambda_{1} \phi^{2}-\mu^{2}\right) A^{a \mu}=\frac{g \hbar c}{2} \bar{\psi} \gamma^{\mu} \sigma^{a} \psi, \\
\square \phi-\lambda_{1} A_{\mu}^{a} A^{a \mu} \phi-\lambda_{2} \phi\left(M^{2}-\phi^{2}\right)=\frac{\Lambda}{2} g \hbar(\bar{\psi} \psi)^{2}, \\
i \hbar \gamma^{\mu}\left(\partial_{\mu}-i \frac{g}{2} \sigma^{a} A_{\mu}^{a}\right) \psi+\Lambda g \hbar \phi \psi(\bar{\psi} \psi)-m_{f} c \psi=0 .
\end{gathered}
$$

Let us note the following features of this set of equations: (i) the presence of the term $\left(\lambda_{1} \phi^{2}-\mu^{2}\right)$ in Eq. (2) will lead to exponential damping of the SU(2) Proca field, and this is a distinctive feature of the Proca monopole compared with the 't Hooft-Polyakov monopole; (ii) the Higgs field $\phi$ is topologically trivial, and this is also the distinction of principle compared with the 't Hooft-Polyakov monopole; (iii) the nonlinear spinor field $\psi$ allows the existence of particlelike solutions even in the absence of the fields $A_{\mu}^{a}$ and $\phi$ (see Refs. [11,12]); (iv) the system supported by the fields $A_{\mu}^{a}$ and $\phi$ also allows the existence of particlelike solutions, a Proca monopole; (v) particlelike solutions exist only for some special choices of the constants $\mu, M$, and $u_{1}$ [for the definition of parameter $u_{1}$, see Eq. (21)]; and (vi) in the absence of the fields $A_{\mu}^{a}$ and $\phi$, the nonlinear Dirac equation (4) describes a system with a mass gap (see Refs. [11,12]), in contrast to Eqs. (2) and (3), which yield a system whose energy spectrum has no mass gap.

To obtain particlelike solutions, the above equations will be solved numerically since apparently it is impossible to find their analytical solution. As will be shown below, these particlelike solutions have the following interesting feature. For each central value of the scalar field $\phi_{0}$ the plane $\left\{f_{2}, E\right\}$ [where $E / \hbar$ is the spinor frequency, see Eq. (8), and $f_{2}$ is a free parameter associated with the Proca field, see Eq. (19)] is divided into two regions: in one of them, particlelike solutions do exist, and in the other one they are absent. This means that there is some curve $\gamma$ dividing these two regions. Near this curve there is a complicated interaction between the fields $A_{\mu}^{a}, \phi$, and $\psi$, which possibly results in an infinite energy of the particlelike solution on the curve $\gamma$.

\section{EQUATIONS FOR A PROCA MONOPOLE INTERACTING WITH NONLINEAR HIGGS AND DIRAC FIELDS}

We seek particlelike solutions describing objects supported by a radial magnetic field (a Proca monopole or a 
Proca hedgehog) and a nonlinear spinor field. For this purpose, we employ the standard SU(2) monopole Ansatz (5) and the Ansatz (8) for the spinor field, each row of which describes a spinor for the ground state of an electron in a hydrogen atom; i.e., we seek a solution of Eqs. (2)-(4) in the following form:

$$
\begin{gathered}
A_{i}^{a}=-\frac{1}{g}[1-f(r)]\left(\begin{array}{ccc}
0 & \sin \varphi & \sin \theta \cos \theta \cos \varphi \\
0 & \cos \varphi & -\sin \theta \cos \theta \sin \varphi \\
0 & 0 & -\sin ^{2} \theta
\end{array}\right), \\
i=r, \theta, \varphi \text { (in polar coordinates), } \\
A_{t}^{a}=0, \\
\phi=\frac{\xi(r)}{g}, \\
\psi^{T}=\frac{e^{-i \frac{E t}{\hbar}}}{g r \sqrt{2}\left\{\left(\begin{array}{c}
0 \\
-u
\end{array}\right),\left(\begin{array}{c}
u \\
0
\end{array}\right),\left(\begin{array}{c}
i v \sin \theta e^{-i \varphi} \\
-i v \cos \theta
\end{array}\right),\right.} \\
\left.\left(\begin{array}{c}
-i v \cos \theta \\
-i v \sin \theta e^{i \varphi}
\end{array}\right)\right\},
\end{gathered}
$$

where $E / \hbar$ is the spinor frequency and the color index $a=1,2,3$. The functions $u$ and $v$ depend on the radial coordinate $r$ only. The Ansatz (8) is taken from Refs. [13,14]. After substituting expressions (5)-(8) into the Lagrangian (1), we have

$$
\begin{aligned}
\tilde{\mathcal{L}}_{\text {eff }} \equiv & \frac{\mathcal{L}_{\text {eff }}}{\hbar c / r_{0}^{4}} \\
= & \frac{1}{\tilde{g}^{2}}\left\{-\left(\frac{f^{\prime 2}}{x^{2}}+\frac{\left(f^{2}-1\right)^{2}}{2 x^{4}}-\tilde{\mu}^{2} \frac{(f-1)^{2}}{x^{2}}\right)\right. \\
& \left.-2 m^{2}\left[\tilde{\xi}^{\prime 2}+\tilde{\lambda}_{1} \frac{(f-1)^{2}}{2 x^{2}} \tilde{\xi}^{2}+\frac{\tilde{\lambda}}{2}\left(\tilde{\xi}^{2}-\tilde{M}^{2}\right)^{2}\right]\right\} \\
& +\frac{1}{x^{2}}\left[-\tilde{u} \tilde{v}^{\prime}+\tilde{u}^{\prime} \tilde{v}-2 f \frac{\tilde{u} \tilde{v}}{x}+m^{2} \frac{\tilde{\Lambda}}{2} \tilde{\xi} \frac{\left(\tilde{u}^{2}-\tilde{v}^{2}\right)^{2}}{x^{2}}\right. \\
& \left.-\tilde{m}_{f}\left(\tilde{u}^{2}-\tilde{v}^{2}\right)+\tilde{E}\left(\tilde{u}^{2}+\tilde{v}^{2}\right)\right] .
\end{aligned}
$$

Here, for the convenience of performing numerical calculations, we have introduced the following dimensionless variables: $\tilde{g}^{2}=g^{2} \hbar c$ is the dimensionless coupling constant for the SU(2) Proca gauge field; $x=r / r_{0}$, where $r_{0}$ is a constant corresponding to the characteristic size of the system under consideration; $\tilde{u}=\sqrt{r_{0}} u / g, \tilde{v}=\sqrt{r_{0}} v / g$, $\tilde{\mu}=r_{0} \mu, \quad \tilde{\xi}=r_{0} g \phi / 2 m, \quad \tilde{M}=g r_{0} M / 2 m, \quad \tilde{\lambda}_{1}=4 \lambda_{1} / g^{2}$, $\tilde{\lambda}=4 m^{2} \lambda_{2} / g^{2}, \quad \tilde{m}_{f}=r_{0} m_{f} c / \hbar, \quad \tilde{E}=r_{0} E /(\hbar c), \quad \tilde{\Lambda}=$ $2 \Lambda /\left(m r_{0}^{3}\right) ; m$ is a free parameter introduced for convenience; and the prime denotes differentiation with respect to $x$. The parameter $r_{0}$ must depend only on constants of a theory; therefore it can be taken, for instance, in the form $r_{0}=\alpha \hbar /\left(m_{f} c\right)$, where $\alpha$ is a constant.

Equations for the unknown functions $f(x), \xi(x), u(x)$, and $v(x)$ can be obtained either by substituting Eqs. (5)-(8) into the field equations, (2)-(4), or by varying the Lagrangian (9) with respect to the corresponding functions $f(x), \xi(x), u(x)$, and $v(x)$. However, in the latter case one should take into account that the nonlinear term $(\bar{\psi} \psi)^{2}=$ $\frac{\left(\tilde{u}^{2}-\tilde{u}^{2}\right)^{2}}{x^{4}}$ must be written in the form $\frac{\left(\tilde{u}_{\psi}^{2}-\tilde{v}_{\psi}^{2}\right)\left(\tilde{u}_{\tilde{\psi}}^{2}-\tilde{v}_{\tilde{\psi}}^{2}\right)}{x^{4}}$, where $(\tilde{u}, \tilde{v})_{\bar{\psi}}$ are taken from $\bar{\psi}$ and $(u, v)_{\psi}$-from $\psi$. Therefore, the corresponding Dirac equation is obtained by varying (9) with respect to $(\tilde{u}, \tilde{v})_{\bar{\psi}}$, and then one must take $(\tilde{u}, \tilde{v})_{\bar{\psi}}=(\tilde{u}, \tilde{v})_{\psi}=(\tilde{u}, \tilde{v})$. Thus, we obtain the following equations:

$$
-f^{\prime \prime}+\frac{f\left(f^{2}-1\right)}{x^{2}}-m^{2}(1-f) \tilde{\xi}^{2}+\tilde{g}^{2} \frac{\tilde{u} \tilde{v}}{x}=-\tilde{\mu}^{2}(1-f),
$$

$$
\tilde{\xi}^{\prime \prime}+\frac{2}{x} \tilde{\xi}^{\prime}=\tilde{\xi}\left[\frac{(1-f)^{2}}{2 x^{2}}+\tilde{\lambda}\left(\tilde{\xi}^{2}-\tilde{M}^{2}\right)\right]-\frac{\tilde{g}^{2} \tilde{\Lambda}}{8} \frac{\left(\tilde{u}^{2}-\tilde{v}^{2}\right)^{2}}{x^{4}}
$$

$$
\begin{aligned}
& \tilde{v}^{\prime}+\frac{f \tilde{v}}{x}=\tilde{u}\left(-\tilde{m}_{f}+\tilde{E}+m^{2} \tilde{\Lambda} \frac{\tilde{u}^{2}-\tilde{v}^{2}}{x^{2}} \tilde{\xi}\right), \\
& \tilde{u}^{\prime}-\frac{f \tilde{u}}{x}=\tilde{v}\left(-\tilde{m}_{f}-\tilde{E}+m^{2} \tilde{\Lambda} \frac{\tilde{u}^{2}-\tilde{v}^{2}}{x^{2}} \tilde{\xi}\right) .
\end{aligned}
$$

For the convenience of performing numerical calculations, we have taken $\tilde{\lambda}_{1}=1$. Equations (10) and (11) describe the Proca monopole with the sources appearing due to the presence of the Dirac and Higgs fields. We emphasize that these equations differ considerably from those describing the 't Hooft-Polyakov monopole. Equations (12) and (13) have been studied in a simplified form for the case of $f=1$ and $\tilde{\xi}=$ const in Refs. [11,12], where it was shown that this set of equations has particlelike solutions whose energy spectrum possesses a mass gap (for brevity, we will refer to such solution as a spinball).

Next, by definition, the energy density of the spinor field is

$$
\epsilon_{s}=i \hbar \bar{\zeta} \gamma^{0} \dot{\zeta}-L_{D, \text { eff }}
$$

where the dot denotes differentiation with respect to time. The Lagrangian of the Dirac field $L_{D \text {,eff }}$ appearing here is given by the expression from (9), 


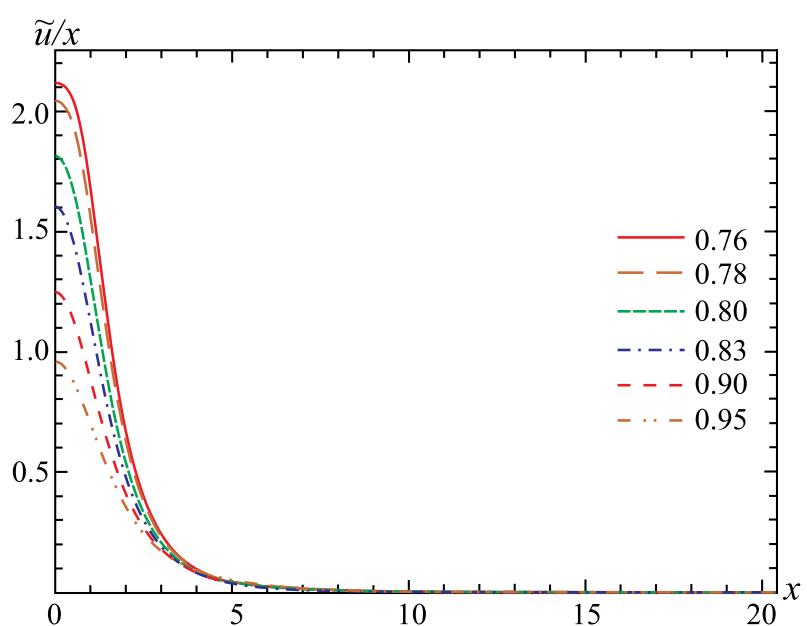

(a)

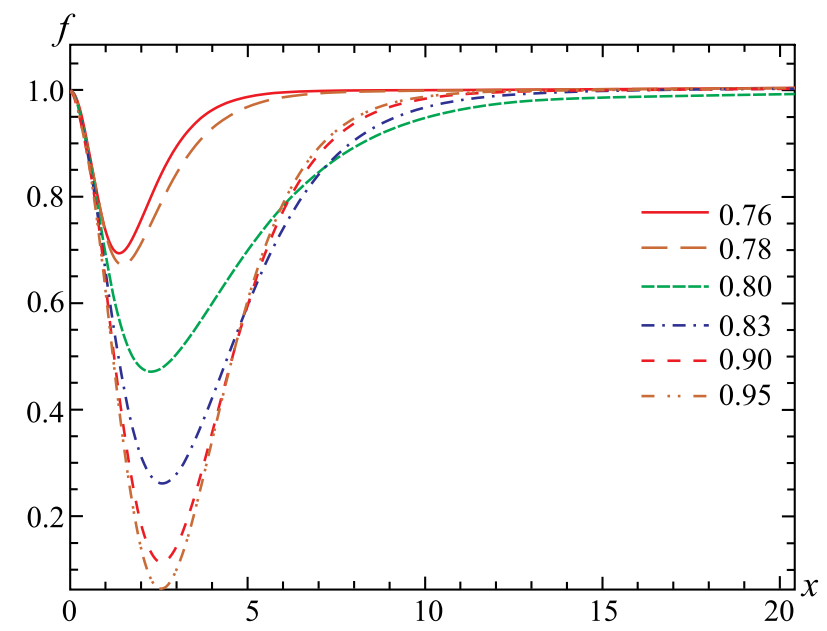

(c)

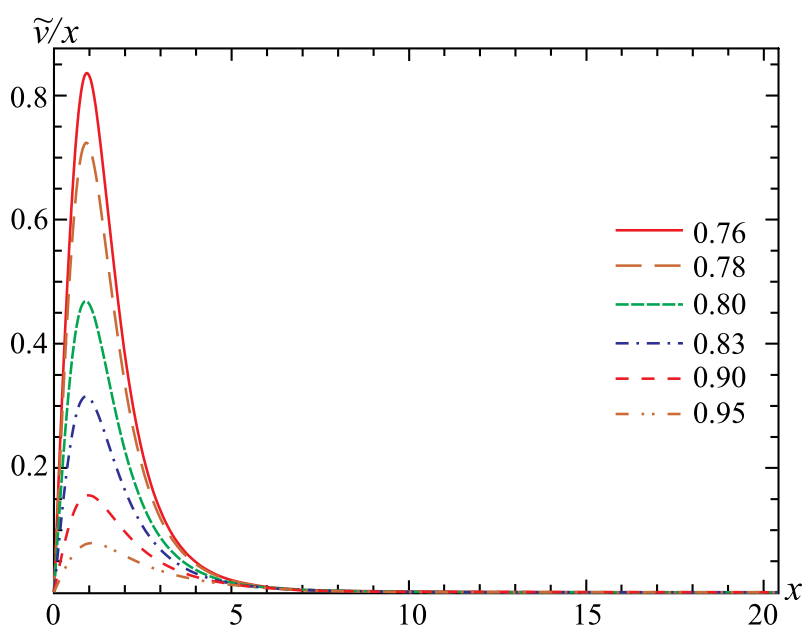

(b)

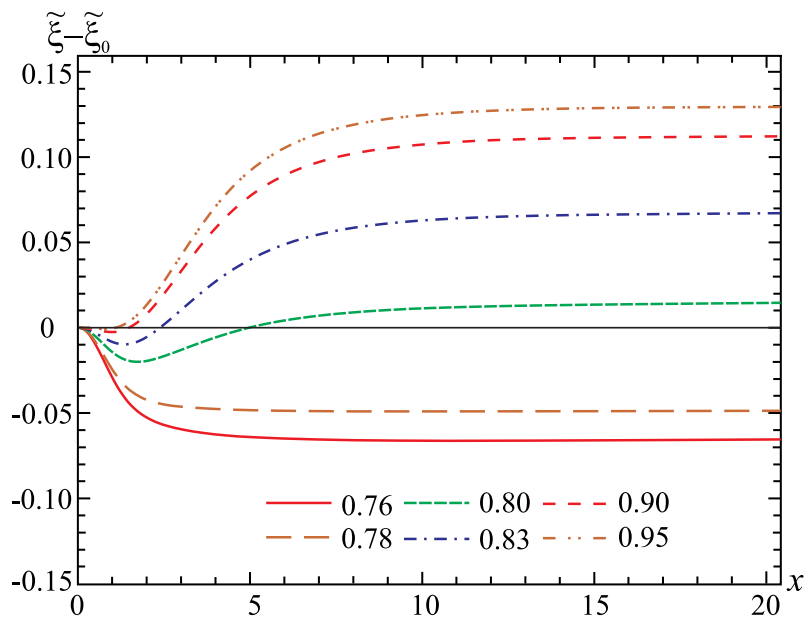

(d)

FIG. 1. Families of the particlelike solutions for $\tilde{\xi}_{0}=0.5$ and $f_{2}=-0.95$ for the Proca-monopole-plus-spinball system with $\tilde{\Lambda}=1 / 9$, $\tilde{m}_{f}=1, m=3, \tilde{g}=1, \tilde{\lambda}=0.1$ for different values of the parameter $\tilde{E}$ (designated by the numbers near the curves). (a) The function $\tilde{u}(x) / x$. (b) The function $\tilde{v}(x) / x$. (c) The function $f(x)$. (d) The function $\tilde{\xi}(x)-\tilde{\xi}_{0}$.

$$
\begin{aligned}
\tilde{L}_{D, \mathrm{eff}}= & \frac{1}{x^{2}}\left[-\tilde{u} \tilde{v}^{\prime}+\tilde{u}^{\prime} \tilde{v}-2 f \frac{\tilde{u} \tilde{v}}{x}+m^{2} \frac{\tilde{\Lambda}}{2} \tilde{\xi} \frac{\left(\tilde{u}^{2}-\tilde{v}^{2}\right)^{2}}{x^{2}}\right. \\
& \left.-\tilde{m}_{f}\left(\tilde{u}^{2}-\tilde{v}^{2}\right)+\tilde{E}\left(\tilde{u}^{2}+\tilde{v}^{2}\right)\right] \\
= & -m^{2} \frac{\tilde{\Lambda}}{2} \tilde{\xi} \frac{\left(\tilde{u}^{2}-\tilde{v}^{2}\right)^{2}}{x^{4}},
\end{aligned}
$$

which is obtained using Eqs. (12) and (13). Then, using the Ansatz (8), the energy density of the spinor field (14) can be found in the following dimensionless form:

$$
\tilde{\epsilon}_{s}=\tilde{E} \frac{\tilde{u}^{2}+\tilde{v}^{2}}{x^{2}}+m^{2} \frac{\tilde{\Lambda}}{2} \tilde{\xi} \frac{\left(\tilde{u}^{2}-\tilde{v}^{2}\right)^{2}}{x^{4}} .
$$

As a result, we get the following total energy density of the particlelike solution:

$$
\tilde{\epsilon}=\tilde{\epsilon}_{\mathrm{Pm}}+\tilde{\epsilon}_{s},
$$

where

$$
\begin{aligned}
\tilde{\epsilon}_{\mathrm{Pm}}= & \frac{1}{\tilde{g}^{2}}\left\{\left[\frac{f^{\prime 2}}{x^{2}}+\frac{\left(f^{2}-1\right)^{2}}{2 x^{4}}-\tilde{\mu}^{2} \frac{(f-1)^{2}}{x^{2}}\right]\right. \\
& \left.+2 m^{2}\left[\tilde{\xi}^{\prime 2}+\frac{(f-1)^{2}}{2 x^{2}} \tilde{\xi}^{2}+\frac{\tilde{\lambda}}{2}\left(\tilde{\xi}^{2}-\tilde{M}^{2}\right)^{2}\right]\right\}
\end{aligned}
$$

is the energy of the Proca monopole. The formula (17) is remarkable because the total energy of the particlelike solution is a sum of energies of the Proca monopole and of the spinball, despite the strong interaction between the fields $f$ and $\xi$, which make up the Proca monopole, and the fields $u$ and $v$ supporting the spinball. 


\section{PARTICLELIKE SOLUTIONS: A PROCA MONOPOLE PLUS A SPINBALL}

This section is devoted to studying particlelike solutions of Eqs. (10)-(13). Since apparently there is no analytical solution of this set of equations, we seek a numerical solution. Because of the presence of terms containing $x$ in the denominators of Eqs. (10)-(13), to perform numerical computations, we assign boundary conditions near the origin $x=0$ where solutions are sought in the form of the Taylor series

$$
\begin{gathered}
f=1+\frac{f_{2}}{2} x^{2}+\ldots, \\
\tilde{\xi}=\tilde{\xi}_{0}+\frac{\tilde{\xi}_{2}}{2} x^{2}+\ldots, \quad \text { where } \\
\tilde{\xi}_{2}=-\frac{\tilde{g}^{2} \tilde{\Lambda} \tilde{u}_{1}^{4}}{24}-\frac{\tilde{\lambda} \tilde{\xi}_{0}}{3}\left(\tilde{\xi}_{0}^{2}-\tilde{M}^{2}\right),
\end{gathered}
$$

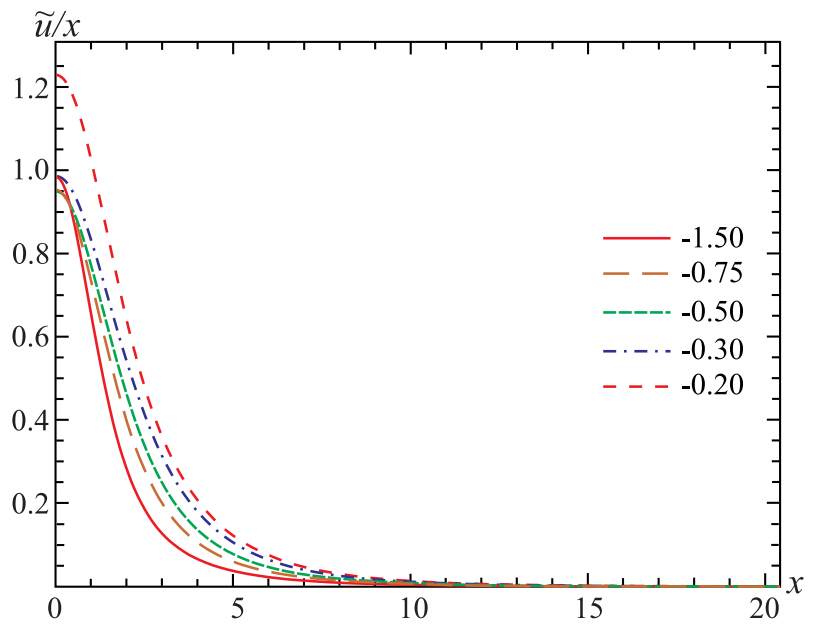

(a)

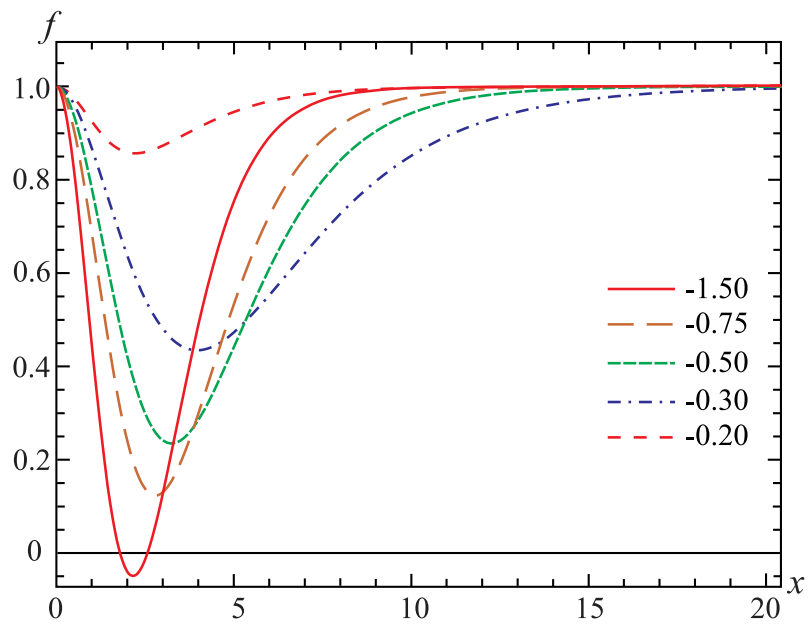

(c)

$$
\tilde{u}=\tilde{u}_{1} x+\frac{\tilde{u}_{3}}{3 !} x^{3}+\ldots
$$

$$
\begin{aligned}
\tilde{v} & =\frac{\tilde{v}_{2}}{2} x^{2}+\frac{\tilde{v}_{4}}{4 !} x^{4}+\ldots, \quad \text { where } \\
\tilde{v}_{2} & =\frac{2}{3} \tilde{u}_{1}\left(\tilde{E}-\tilde{m}_{f}+m^{2} \tilde{\Lambda} \tilde{\xi}_{0} \tilde{u}_{1}^{2}\right) .
\end{aligned}
$$

The expansion coefficients $f_{2}, \tilde{\xi}_{0}$, and $\tilde{u}_{1}$ appearing here are free parameters whose values cannot be found from Eqs. (10)-(13).

Equations (10)-(13) are solved numerically as a nonlinear problem for the eigenvalues $\tilde{\mu}, \tilde{M}$, and $\tilde{u}_{1}$ and for the eigenfunctions $f, \tilde{\xi}$, $\tilde{v}$, and $\tilde{u}$. Figure 1 depicts a typical behavior of the solutions for fixed values of $\tilde{\xi}_{0}$ and $f_{2}$, Fig. 2, for fixed values of $\tilde{\xi}_{0}$ and $\tilde{E}$, and Fig. 3, for fixed

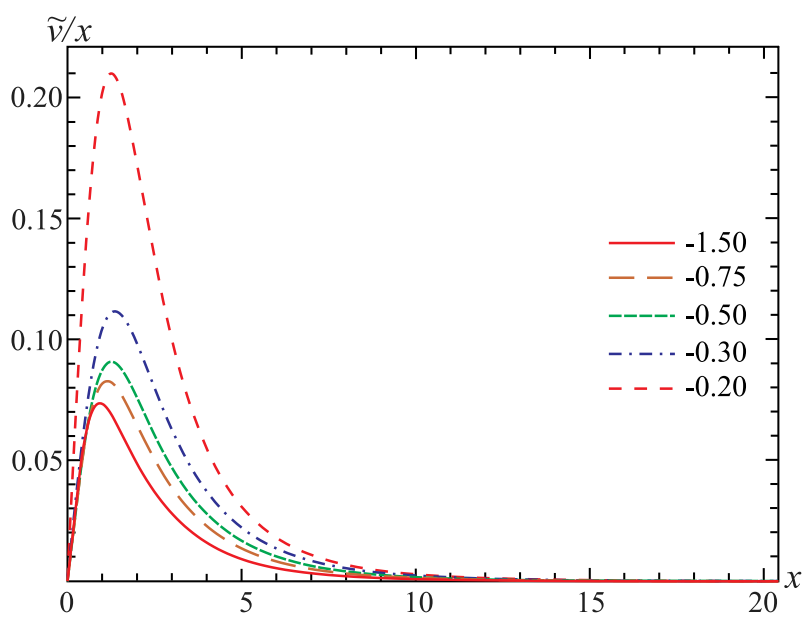

(b)

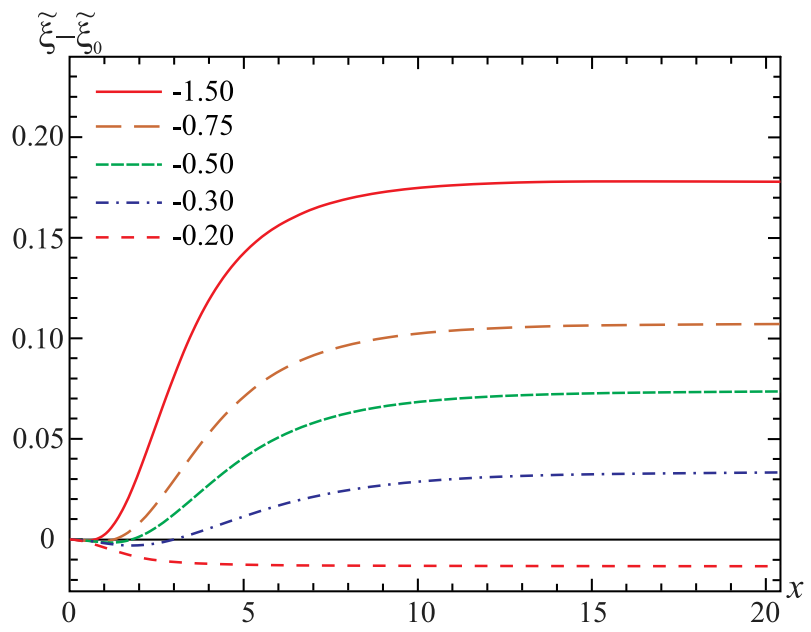

(d)

FIG. 2. Families of the particlelike solutions for $\tilde{\xi}_{0}=0.5$ and $\tilde{E}=0.95$ for the Proca-monopole-plus-spinball system with $\tilde{\Lambda}=1 / 9$, $\tilde{m}_{f}=1, m=3, \tilde{g}=1, \tilde{\lambda}=0.1$ for different values of the parameter $f_{2}$ (designated by the numbers near the curves). (a) The function $\tilde{u}(x) / x$. (b) The function $\tilde{v}(x) / x$. (c) The function $f(x)$. (d) The function $\tilde{\xi}(x)-\tilde{\xi}_{0}$. 
values of $f_{2}$ and $\tilde{E}$. Figure 4 shows the eigenvalues $\tilde{\mu}, \tilde{M}$, and $\tilde{u}_{1}$ as functions of the parameters $f_{2}, \tilde{E}$.

The asymptotic behavior of the solutions as $x \rightarrow \infty$ is

$f(x) \approx 1-f_{\infty} e^{-x \sqrt{m^{2} \tilde{M}^{2}-\tilde{\mu}^{2}}}-\frac{g^{2} u_{\infty} v_{\infty}}{4 \tilde{m}_{f}^{2}-4 \tilde{E}^{2}+m^{2} \tilde{M}^{2}-\tilde{\mu}^{2}}$

$\times \frac{e^{-2 x \sqrt{\tilde{m}_{f}^{2}-\tilde{E}^{2}}}}{x}$,

$\tilde{\xi}(x) \approx \tilde{M}-\delta \tilde{\xi}, \quad \tilde{u}(x) \approx \tilde{u}_{\infty} e^{-x \sqrt{\tilde{m}_{f}^{2}-\tilde{E}^{2}}}$,

$\tilde{v}(x) \approx \tilde{v}_{\infty} e^{-x \sqrt{\tilde{m}_{f}^{2}-\tilde{E}^{2}}}$,

where $f_{\infty}, \tilde{\xi}_{\infty}, \tilde{v}_{\infty}$, and $\tilde{u}_{\infty}$ are integration constants and the function $\delta \tilde{\xi} \ll \tilde{M}$ satisfies the asymptotic equation

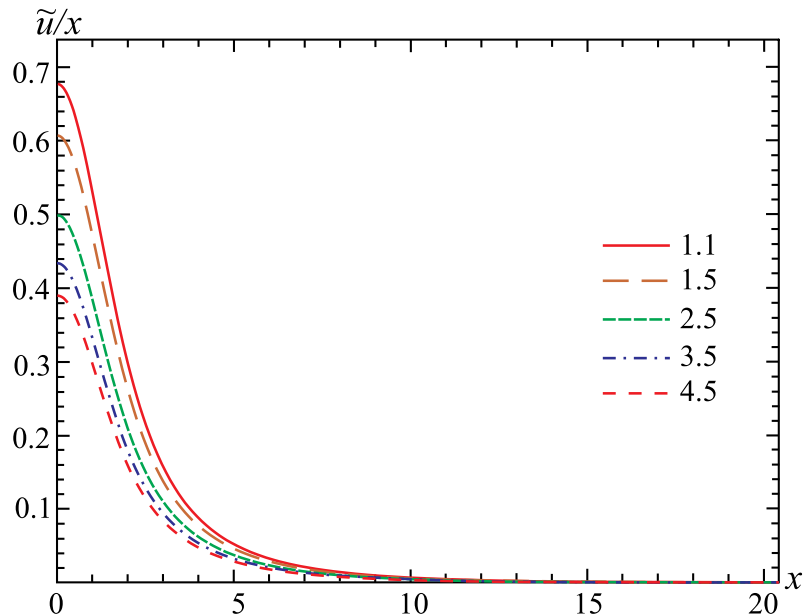

(a)

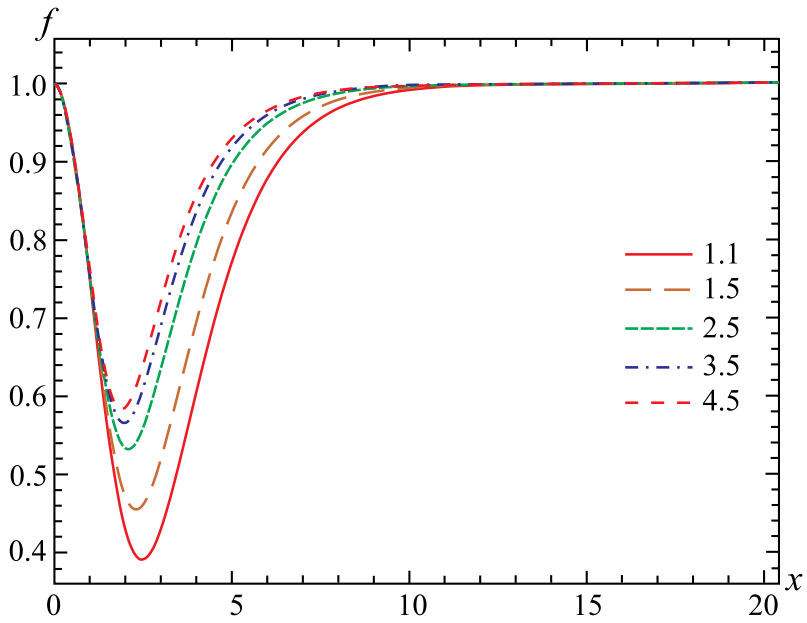

(c)

$$
\begin{aligned}
\delta \tilde{\xi}^{\prime \prime}+\frac{2}{x} \delta \tilde{\xi}^{\prime}= & -\tilde{M}\left(\frac{f_{\infty}^{2} e^{-2 x \sqrt{m^{2} \tilde{M}^{2}-\tilde{\mu}^{2}}}}{2 x^{2}}-2 \tilde{\lambda} \tilde{M} \delta \tilde{\xi}\right) \\
& +\frac{\tilde{g}^{2} \tilde{\Lambda}}{8} \frac{\left(\tilde{u}_{\infty}^{2}-\tilde{v}_{\infty}^{2}\right)^{2}}{x^{4}} e^{-4 x \sqrt{\tilde{m}_{f}^{2}-\tilde{E}^{2}}} .
\end{aligned}
$$

Its solution can be found in the form

$$
\begin{aligned}
\delta \xi= & \xi_{\infty} \frac{e^{-x} \sqrt{2 \tilde{\lambda} \tilde{M}^{2}}}{x}-\frac{\tilde{M} f_{\infty}^{2}}{8\left(m^{2} \tilde{M}^{2}-\tilde{\mu}^{2}\right)} \frac{e^{-2 x \sqrt{m^{2} \tilde{M}^{2}-\tilde{\mu}^{2}}}}{x^{2}} \\
& +\frac{\tilde{g}^{2} \tilde{\Lambda}\left(\tilde{u}_{\infty}^{2}-\tilde{v}_{\infty}^{2}\right)^{2}}{128\left(\tilde{m}_{f}^{2}-\tilde{E}^{2}\right)} \frac{e^{-4 x \sqrt{\tilde{m}_{f}^{2}-\tilde{E}^{2}}}}{x^{4}} .
\end{aligned}
$$

The dimensionless energy density of the system in question can be obtained from Eq. (17). Figure 5 depicts distributions of the energy density for different $f_{2}$ [Fig. 5(a)], $\tilde{E}$ [Fig. 5(b)], and $\tilde{\xi}_{0}$ [Fig. 5(c)].

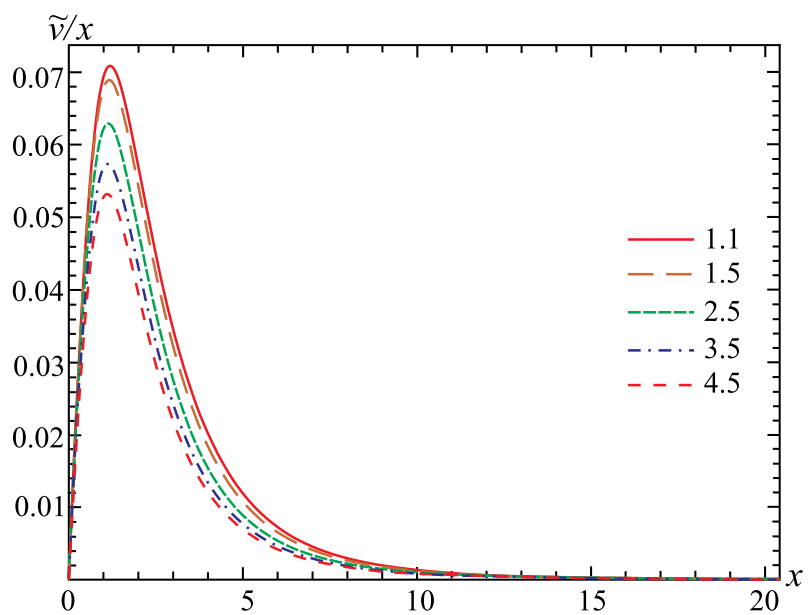

(b)

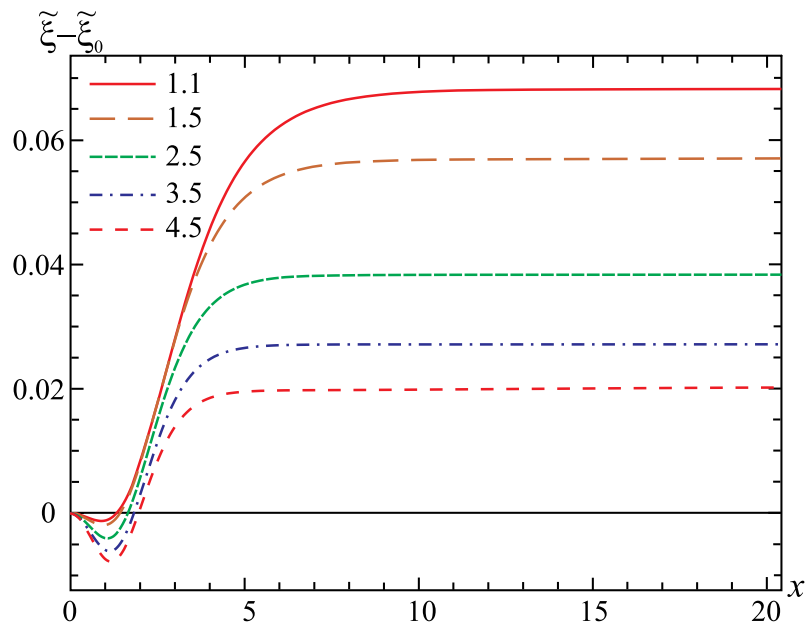

(d)

FIG. 3. Families of the particlelike solutions for $f_{2}=-0.6$ and $\tilde{E}=0.96$ for the Proca-monopole-plus-spinball system with $\tilde{\Lambda}=1 / 9$, $\tilde{m}_{f}=1, m=3, \tilde{g}=1, \tilde{\lambda}=0.1$ for different values of the parameter $\tilde{\xi}_{0}$ (designated by the numbers near the curves). (a) The function $\tilde{u}(x) / x$. (b) The function $\tilde{v}(x) / x$. (c) The function $f(x)$. (d) The function $\tilde{\xi}(x)-\tilde{\xi}_{0}$. 


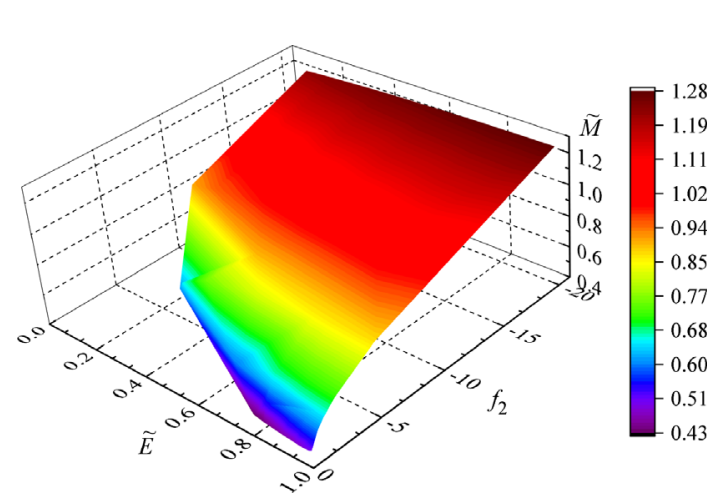

(a)

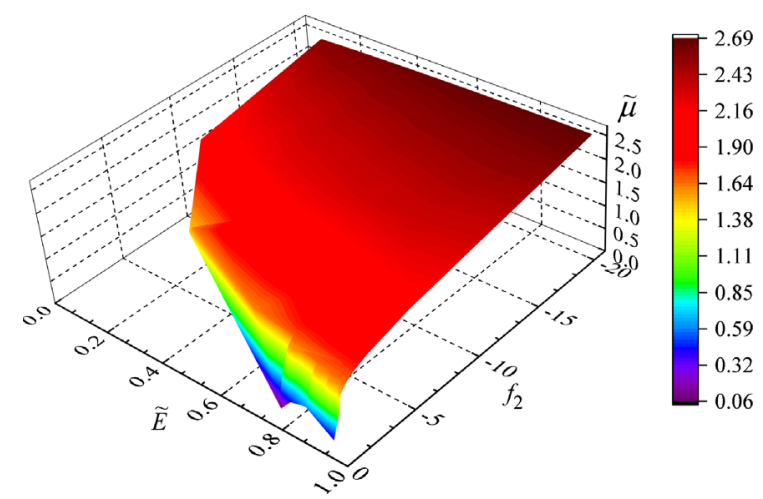

(b)

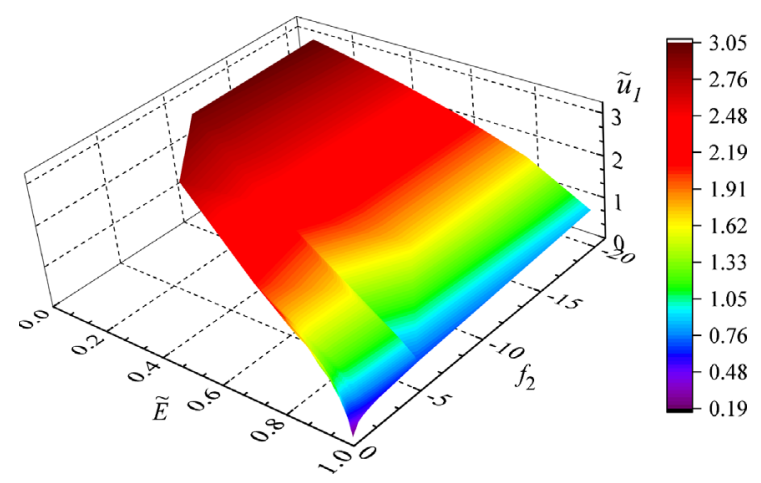

(c)

FIG. 4. The eigenvalues $\tilde{M}, \tilde{\mu}$ and $\tilde{u}_{1}$ as functions of the parameters $f_{2}$ and $\tilde{E}$ for $\tilde{\xi}=0.5$ for the Proca-monopole-plus-spinball system with $\tilde{\Lambda}=1 / 9, \tilde{m}_{f}=1, m=3, \tilde{g}=1, \tilde{\lambda}=0.1$. (a) The dependence $\tilde{M}\left(f_{2}, \tilde{E}\right)$. (b) The dependence $\tilde{\mu}\left(f_{2}, \tilde{E}\right)$. (c) The dependence $\tilde{\mu}_{1}\left(f_{2}, \tilde{E}\right)$.

It is of great interest to follow the behavior of the "magnetic" Proca field (we have been using the word "magnetic" in quotation marks because the concept of a magnetic field is not quite correct as applied to a Proca field). The radial color "magnetic" field is defined as follows:

$$
H_{r}^{a}=\frac{1-f^{2}}{g r^{2}}
$$

The presence of this field enables us to speak of the Proca monopole. Its asymptotic behavior is

$$
H_{r}^{a}(r) \approx \frac{2}{g} \frac{e^{-\frac{r}{r_{0}} \sqrt{m^{2} \tilde{M}^{2}-\tilde{\mu}^{2}}}}{r^{2}} .
$$

It is seen from this expression that the Proca monopole differs in principle from the 't Hooft-Polyakov monopole by its asymptotic behavior. The graphs for the radial "magnetic" Proca field $H_{r}^{a}$ are given in Fig. 6.

\section{ENERGY SPECTRUM OF THE PARTICLELIKE SOLUTIONS FOR FIXED $\tilde{\xi}_{0}$}

In this section we study the energy spectrum of the particlelike solutions as a function of the tree parameters $f_{2}, \tilde{E}$, and $\tilde{\xi}_{0}$. Each such solution describes a ball consisting of the Dirac, Proca, and Higgs fields. Its structure is described by Eqs. (10)-(13), using which we have constructed the corresponding regular solutions in Sec. IV. Here, we find the energy spectrum of such a system and demonstrate the presence of a mass gap for fixed $\tilde{\xi}_{0}$.

Using the expression for the dimensionless energy density $\tilde{\epsilon}$ from Eq. (17), the dimensionless total energy of the system in question is calculated as

$\tilde{W}_{t} \equiv \frac{W_{t}}{\hbar c / r_{0}}=4 \pi \int_{0}^{\infty} x^{2} \tilde{\epsilon} d x=\left(\tilde{W}_{t}\right)_{\mathrm{Pm}}+\left(\tilde{W}_{t}\right)_{s}$

Here, according to the decomposition (17), we have separated the Proca monopole and spinball energies. Solving the set of equations (10)-(13) numerically, we have computed this energy for different values of $f_{2}, \tilde{E}$, and $\tilde{\xi}_{0}$. Our strategy of studying the energy spectrum is as follows: (i) We obtain energy spectra for different values of $\tilde{\xi}_{0}$. (ii) For the spectra obtained, we show the presence of minimum of the energy, i.e., of the mass gap $\Delta$ for a given $\tilde{\xi}_{0}$, $\Delta\left(\tilde{\xi}_{0}\right)$. (iii) We examine a behavior of $\Delta$ as a function of $\tilde{\xi}_{0}$. 


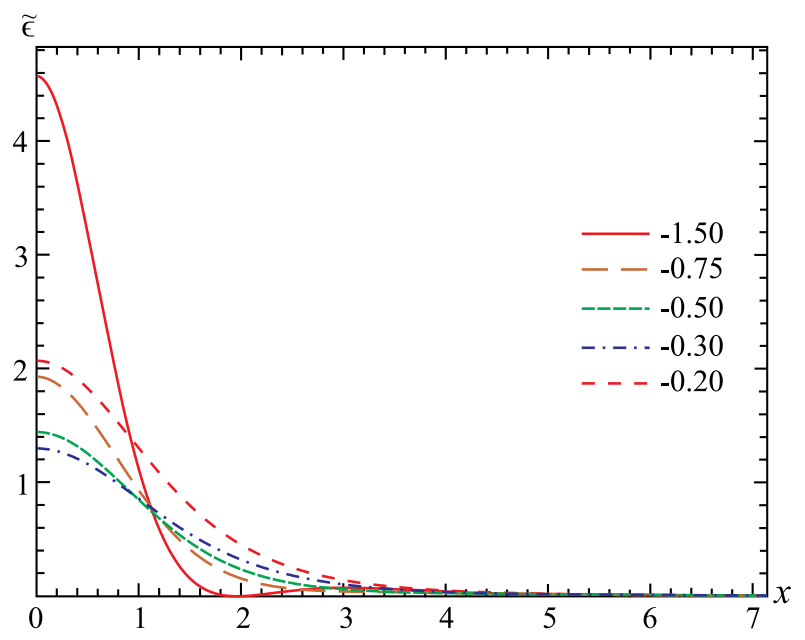

(a)

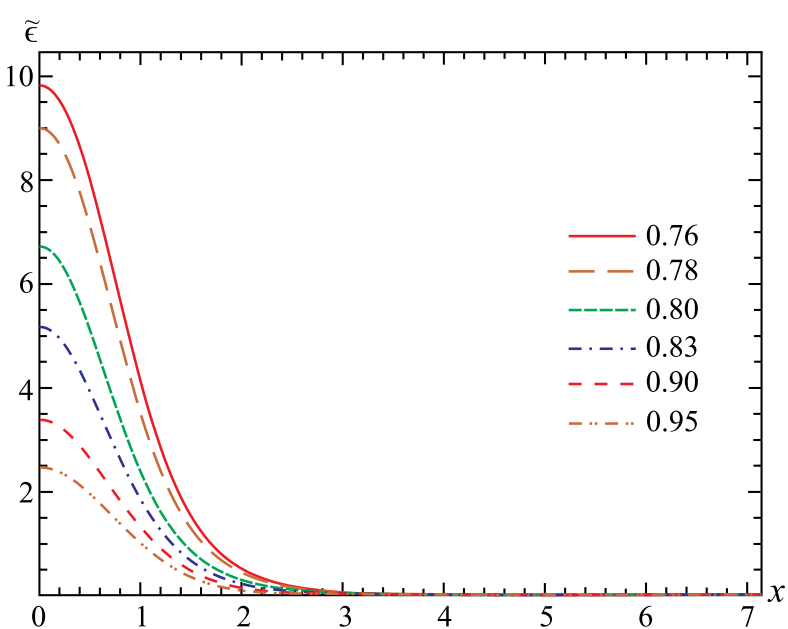

(b)

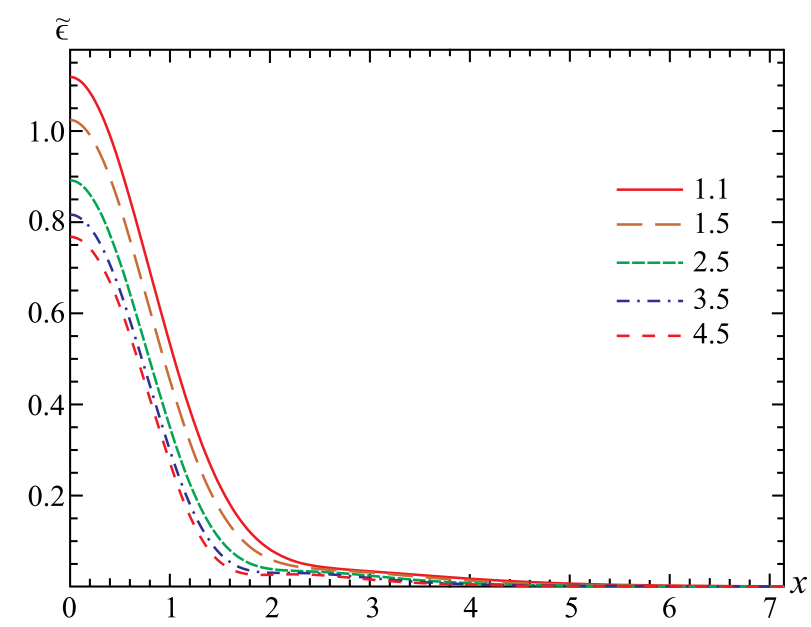

(c)

FIG. 5. The energy density $\tilde{\epsilon}(x)$ of the solution describing the Proca-monopole-plus-spinball system with $\tilde{\Lambda}=1 / 9, \tilde{m}_{f}=1, m=3$, $\tilde{g}=1, \tilde{\lambda}=0.1$ for different values of $f_{2}$ [Fig. 5(a)], $\tilde{E}$ [Fig. 5(b)], and $\tilde{\xi}_{0}$ [Fig. 5(c)]. The corresponding values of $f_{2}, \tilde{E}$, and $\tilde{\xi}_{0}$ are designated by the numbers near the curves. (a) $\tilde{\xi}_{0}=0.5, \tilde{E}=0.95$. (b) $\tilde{\xi}_{0}=0.5, \tilde{E}=-0.95$. (c) $f_{2}=-0.6, \tilde{E}=0.96$.

Three-dimensional and contour plots for the energy (28) are given in Fig. 7. It is seen from Fig. 7(b) that there are closed lines characterizing the presence of extremum of the energy. In the case under consideration, this is a minimum corresponding to the mass gap $\Delta\left(\tilde{\xi}_{0}\right)$ for a given $\tilde{\xi}_{0}$. The approximate value of the dimensionless mass gap for the values of the parameters used here $\left(\tilde{\Lambda}=1 / 9, \tilde{m}_{f}=1\right.$, $\tilde{g}=1$, and $m=3$ ) is

$$
\begin{aligned}
\tilde{\Delta}\left(\tilde{\xi}_{0}=0.8\right) & \equiv \frac{\Delta\left(\tilde{\xi}_{0}=0.8\right)}{\hbar c / r_{0}} \\
& \approx 25.5 \quad \text { for } f_{2} \approx-0.86, \\
\tilde{E} & \approx 0.98 .
\end{aligned}
$$

(For simplicity, below we omit the tilde by $\Delta$.) Similar graphs for the energy of the particlelike solutions can be obtained for other values of $\tilde{\xi}_{0}$ as well. In particular, Fig. 8 illustrates the behavior of the energy (28) in a wide range of values of the parameters $f_{2}$ and $\tilde{E}$ for $\tilde{\xi}_{0}=0.5$.

The numerical computations, as well as Fig. 7, indicate that for any fixed $\tilde{\xi}_{0}$ (at least in the range $0.4<\tilde{\xi}_{0}<4.5$ under investigation) there is a minimum value of the energy, which can be called a mass gap $\Delta\left(\tilde{\xi}_{0}\right)$ for a fixed value of $\tilde{\xi}_{0}$.

Notice here the following important features of the system under consideration:

(i) The particlelike solutions obtained describe objects consisting of a Proca monopole and of a spinball supported by strongly interacting non-Abelian SU(2) Proca vector fields and nonlinear spinor and scalar fields.

(ii) The numerical calculations enable us to speak with certainty that for any fixed value of $\tilde{\xi}_{0}$ there is a minimum value of the energy, which can be called a mass gap $\Delta\left(\tilde{\xi}_{0}\right)$ for fixed $\tilde{\xi}_{0}$; i.e., there is a dependence $\Delta\left(\tilde{\xi}_{0}\right)$. 


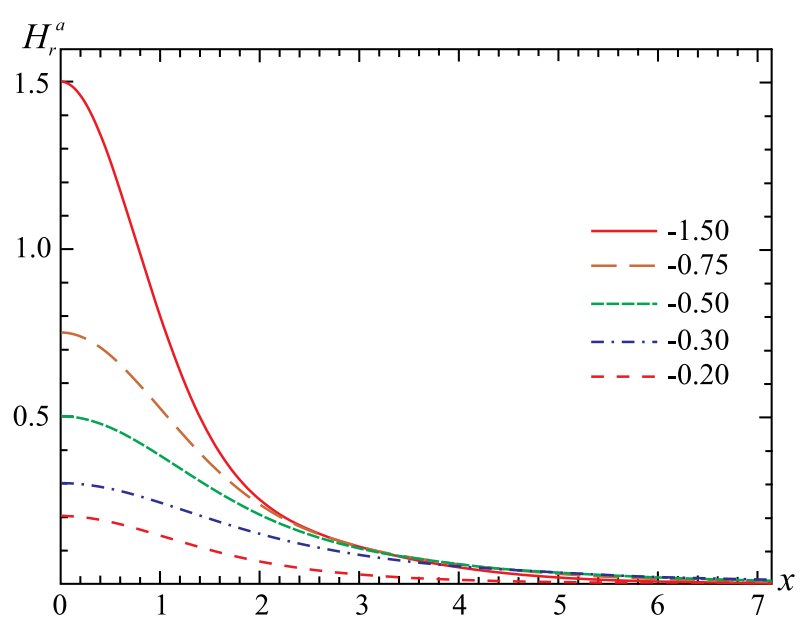

(a)

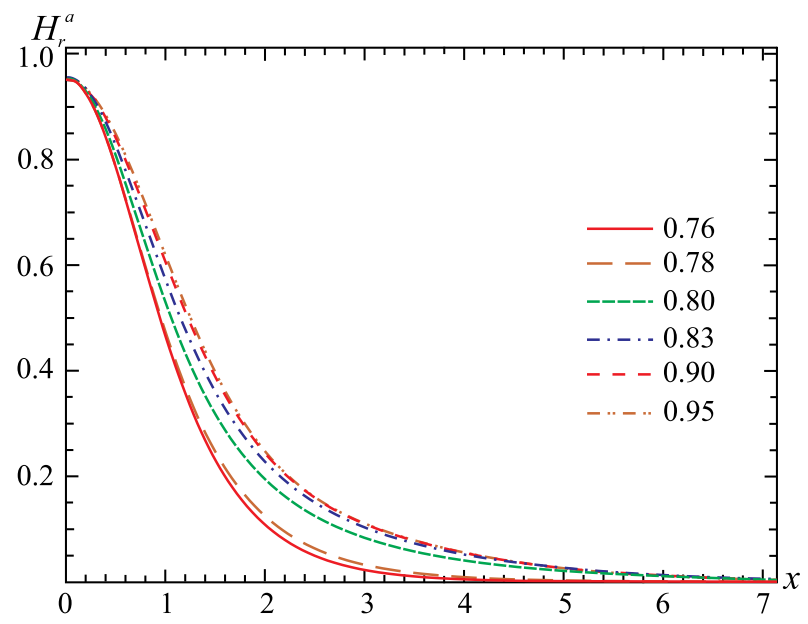

(b)

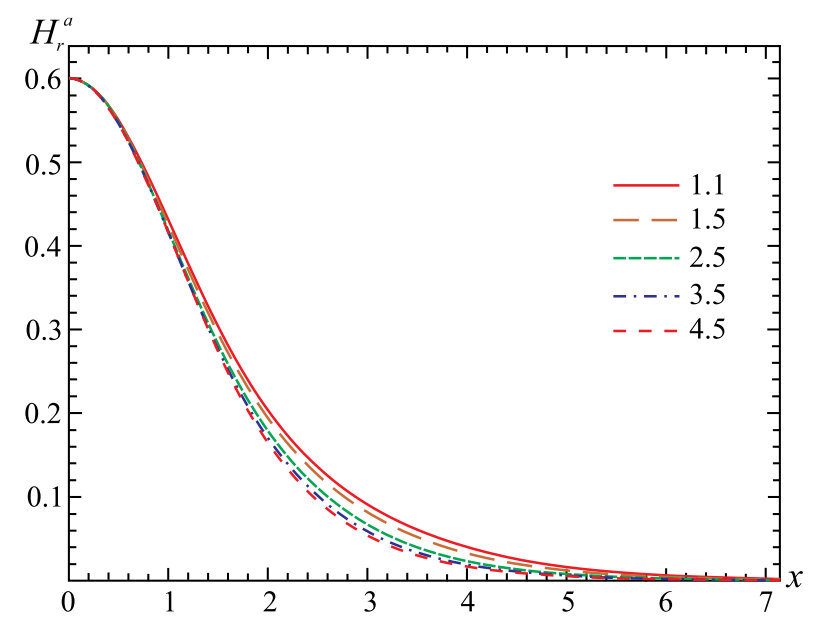

(c)

FIG. 6. The "magnetic" Proca field $H_{r}^{a}(x)$ of the solution describing the Proca-monopole-plus-spinball system with $\tilde{\Lambda}=1 / 9, \tilde{m}_{f}=1$, $m=3, \tilde{g}=1, \tilde{\lambda}=0.1$ for different values of $f_{2}$ [Fig. 6(a)], $\tilde{E}$ [Fig. 6(b)], and $\tilde{\xi}_{0}$ [Fig. 6(c)]. The corresponding values of $f_{2}, \tilde{E}$, and $\tilde{\xi}_{0}$ are designated by the numbers near the curves. (a) $\tilde{\xi}_{0}=1.1, \tilde{E}=0.96$. (b) $\tilde{\xi}_{0}=0.5, f_{2}=-0.95$. (c) $f_{2}=-0.6, \tilde{E}=0.96$.

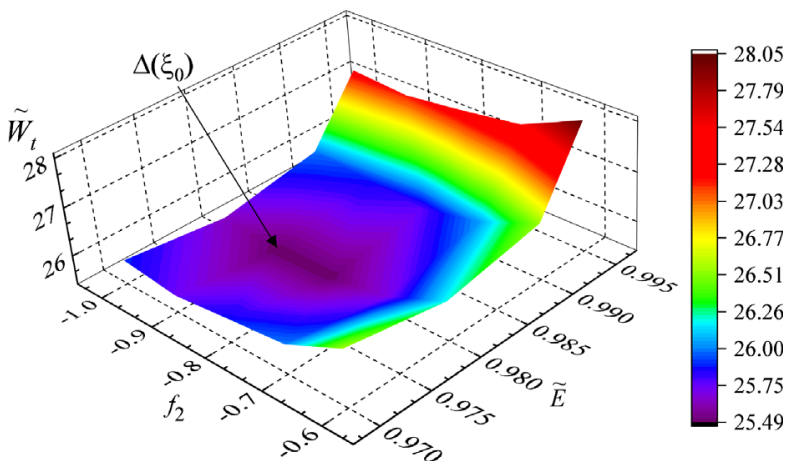

(a)

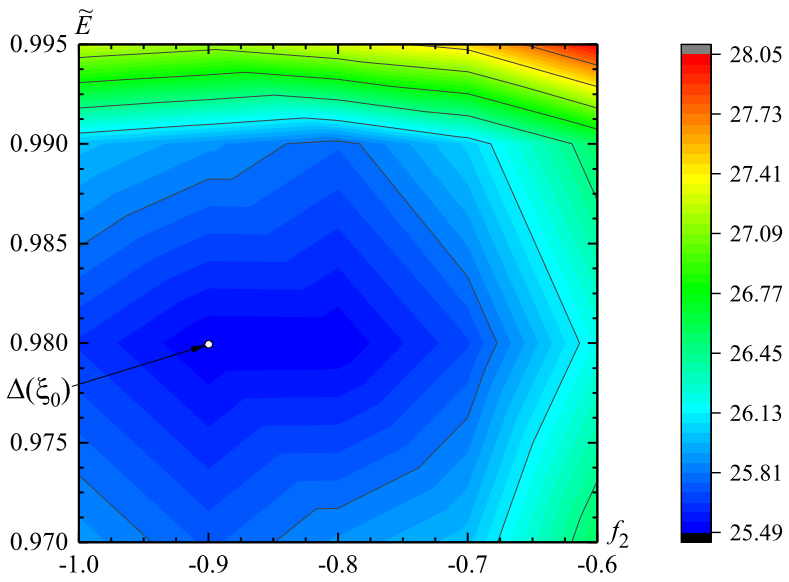

(b)

FIG. 7. (a) $3 \mathrm{D}$ and (b) contour plots of the energy $\tilde{W}_{t}$ from (28) for $\tilde{\xi}_{0}=0.8$ as a function of $f_{2}$ and $\tilde{E}$. 


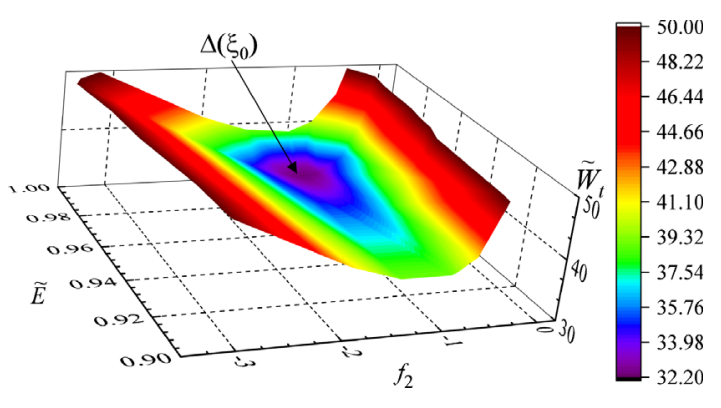

(a)

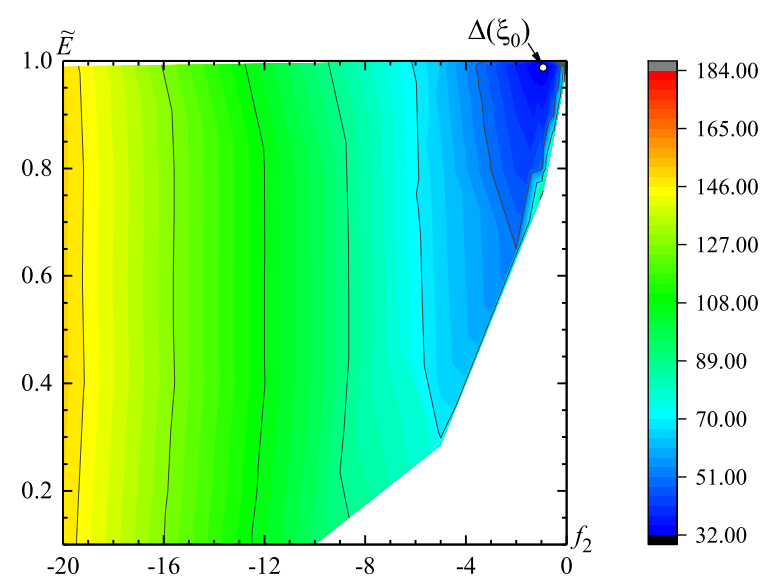

(b)

FIG. 8. (a) 3D and (b) contour plots of the energy $\tilde{W}_{t}$ from (28) for $\tilde{\xi}_{0}=0.5$ as a function of $f_{2}$ and $\tilde{E}$.

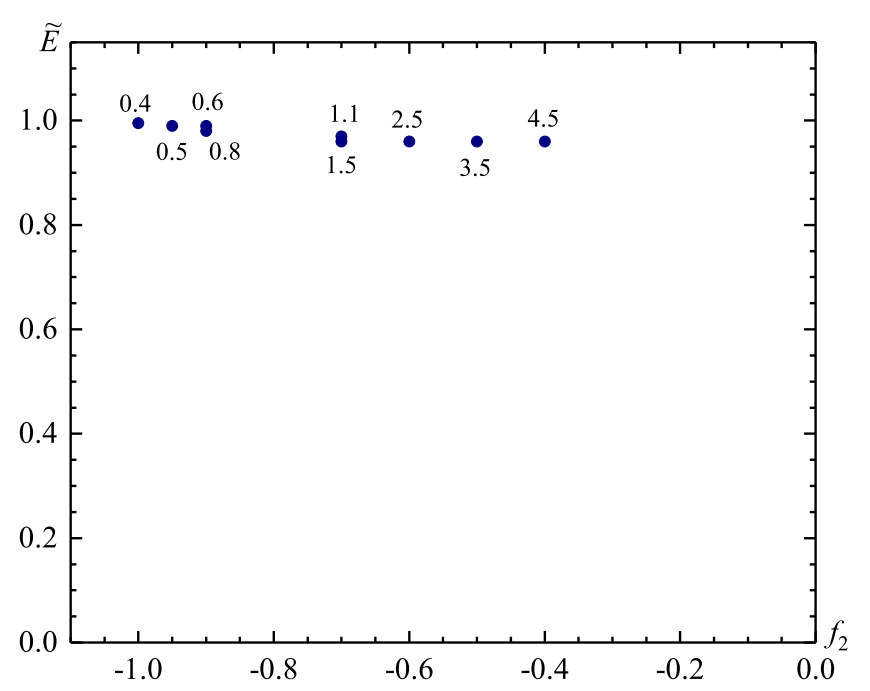

FIG. 9. The positions of minima of the energy $\tilde{W}_{t}$. The numbers near the points denote the corresponding values of $\tilde{\xi}_{0}$.

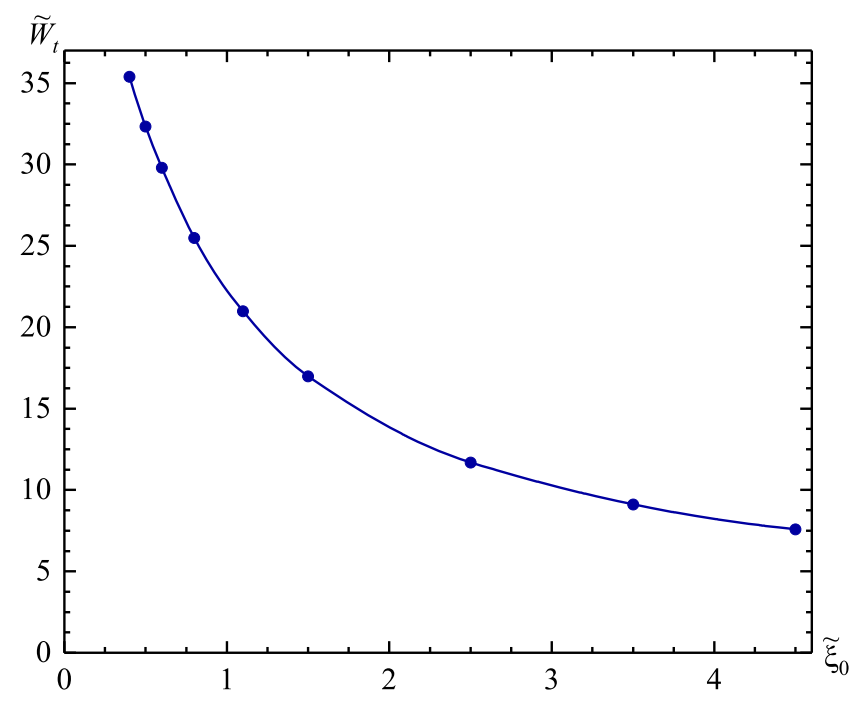

FIG. 10. The minima of the energy $\tilde{W}_{t}$ as a function of $\tilde{\xi}_{0}$. (iii) The numerical analysis does not allow us to determine whether there is a minimum value of the energy (a global value of the mass gap $\Delta$ ) in a whole range of possible values of the parameter $\tilde{\xi}_{0}$. The analysis of the behavior of $\Delta\left(\tilde{\xi}_{0}\right)$ in the range $0.4<\tilde{\xi}_{0}<4.5$ permits us to assume that $\Delta\left(\tilde{\xi}_{0}\right) \stackrel{\tilde{\xi}_{0} \rightarrow \infty}{\rightarrow}\left\{\begin{array}{ll}\text { either } & \text { const } \\ \text { or } & \infty\end{array}\right.$.

(iv) The numerical analysis indicates that as $\tilde{E} \rightarrow 1$, the total energy $\tilde{W}_{t} \rightarrow+\infty$.

(v) Regular solutions of Eqs. (10)-(13) exist not for all pairs $\left\{f_{2}, \tilde{E}\right\}$. It is seen from Fig. 8 that for some fixed $f_{2}$ there exists a critical value $\tilde{E}_{\text {cr }}$ for which the solution still exists but no solutions are found for $\tilde{E}<\tilde{E}_{\text {cr. }}$. According to the numerical calculations, the energy of the solutions obtained tends to infinity when $\tilde{E} \rightarrow \tilde{E}_{\text {cr }}$, i.e., $\tilde{W}_{t} \stackrel{\tilde{E} \rightarrow \tilde{E}_{\text {cr }}}{\longrightarrow}+\infty$.

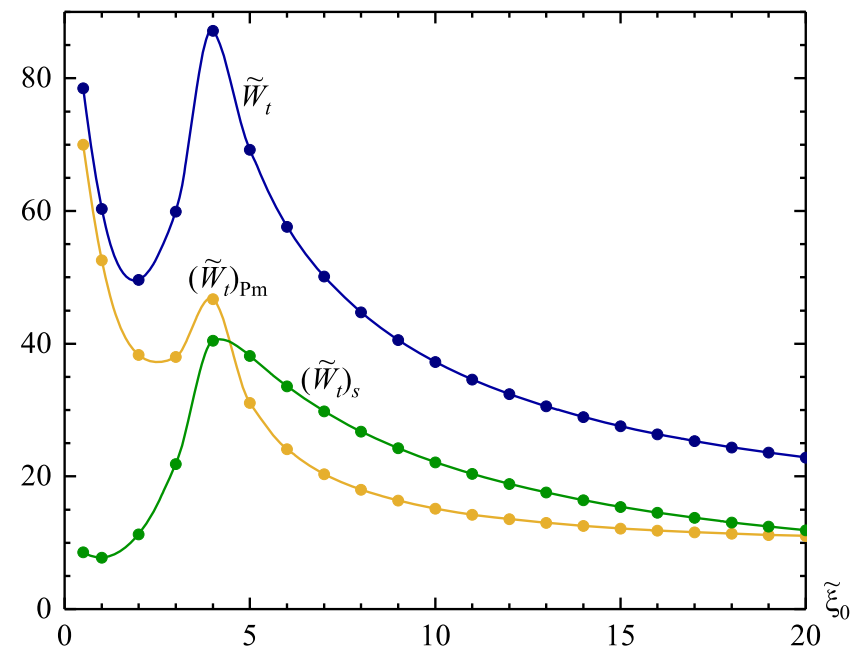

FIG. 11. The energies $\tilde{W}_{t},\left(\tilde{W}_{t}\right)_{\mathrm{Pm}}$, and $\left(\tilde{W}_{t}\right)_{s}$ as functions of the parameter $\tilde{\xi}_{0}$ for $f_{2}=-7$ and $\tilde{E}=0.5$. 


\section{ANALYSIS OF THE BEHAVIOR OF THE MASS} GAP $\Delta\left(\tilde{\xi}_{0}\right)$ FOR DIFFERENT $\tilde{\xi}_{0}$

In Sec. IV, we have shown that the set of equations describing the Proca-Dirac-Higgs system has particlelike Proca-monopole-plus-spinball solutions. In Sec. V, we have studied the energy spectrum of the solutions for fixed values of the parameter $\tilde{\xi}_{0}$ and have shown that the spectrum possesses a mass gap $\Delta\left(\tilde{\xi}_{0}\right)$ for some values of $\tilde{\xi}_{0}$.

The next step is to study the behavior of the mass gap $\Delta\left(\tilde{\xi}_{0}\right)$ in a whole range of values of the parameter $\tilde{\xi}_{0}$. The significance of this problem is due to the fact that if the quantity $\Delta\left(\tilde{\xi}_{0}\right)$ has a global minimum this means that the Proca-Dirac-Higgs theory has a mass gap. The problem of the presence or absence of a mass gap is of great

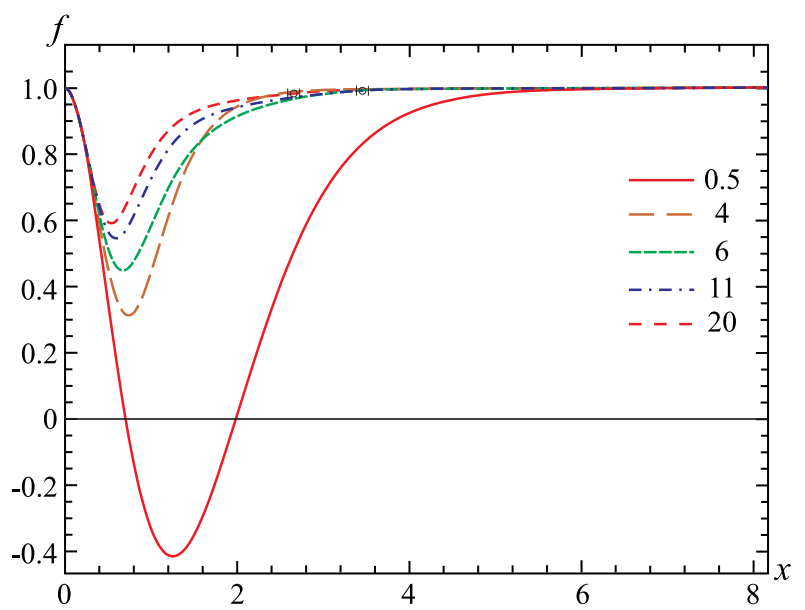

(a)

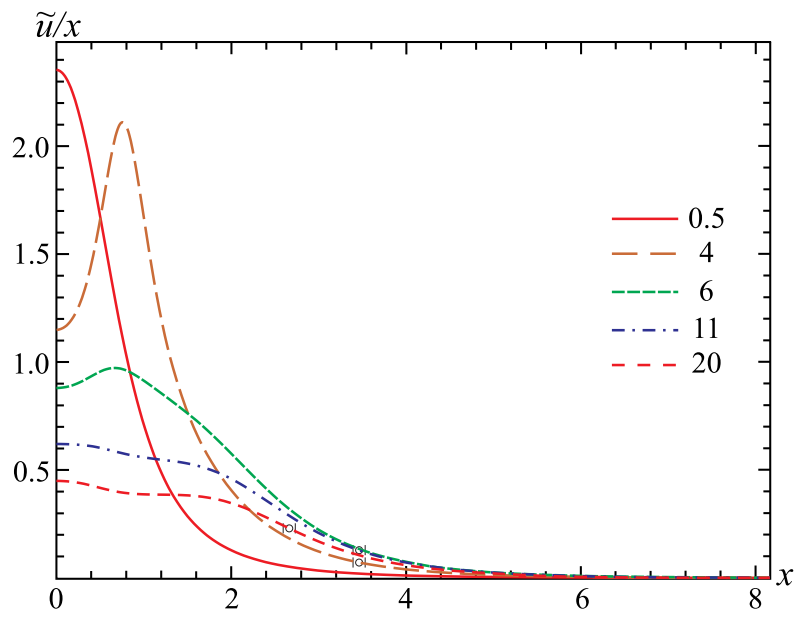

(c) importance in modern quantum field theory. Its solving would practically mean solving the problem of a nonpertubative quantization, and this is necessary, for instance, in constructing a theory of strong interactions. In the present paper we study a classical field theory based on the coupled set of the Proca, nonlinear Klein-Gordon, and Dirac equations. It seems to us that the presence of a mass gap, even in a classical field theory, would be of great interest since it would allow one to get insight into the reason for its appearance.

To study the question of the presence of the mass gap in a whole range of values of the parameters $f_{2}, \tilde{E}$, and $\tilde{\xi}_{0}$, it is necessary to calculate $\Delta\left(\tilde{\xi}_{0}\right)$ for $0<\tilde{\xi}_{0}<\infty$ and to check whether or not there is a global minimum of this function. Figure 10 shows this curve in the range $0.4<\tilde{\xi}_{0}<4.5$.

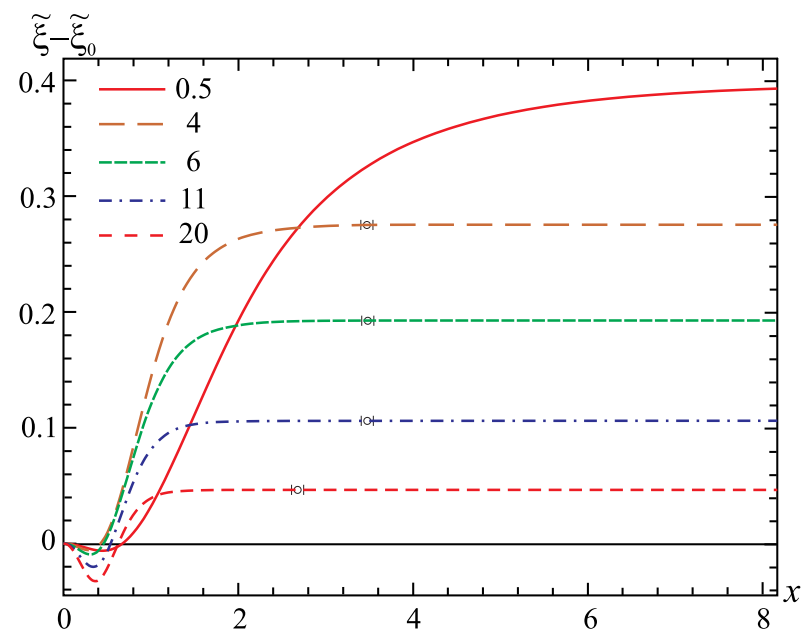

(b)

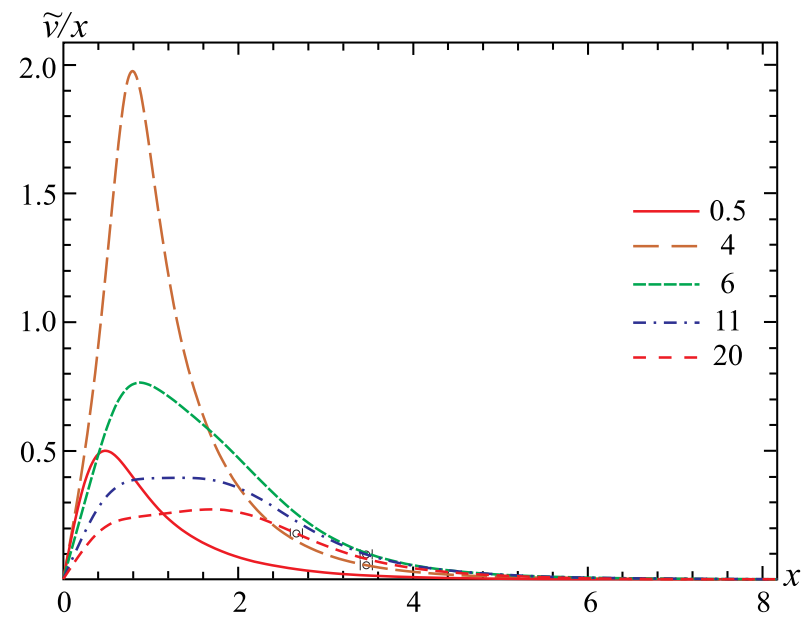

(d)

FIG. 12. Families of particlelike solutions for $f_{2}=-7$ and $\tilde{E}=0.5$ for the Proca-monopole-plus-spinball system with $\tilde{\Lambda}=1 / 9$, $\tilde{m}_{f}=1, m=3, \tilde{g}=1, \tilde{\lambda}=0.1$ for different values of the parameter $\tilde{\xi}_{0}$ (designated by the numbers near the curves). The symbols $|\circ|$ mark the points where (i) in Figs. 12(a) and 12(b), the numerical solutions for the functions $f$ and $\tilde{\xi}$ match with the asymptotic solutions (23) and (25); (ii) in Figs. 12(c) and 12(d), for the functions $\tilde{u}$ and $\tilde{v}$, we sought a numerical solution of only two equations (12) and (13) with replacing the functions $f$ and $\tilde{\xi}$ by the asymptotic expressions (23) and (25). (a) The function $f(x)$. (b) The function $\tilde{\xi}(x)-\tilde{\xi}_{0}$. (c) The function $\tilde{u}(x) / x$. (d) The function $\tilde{v} / x$. 
Unfortunately, deriving solutions for small $f_{2}$ and large $\tilde{\xi}_{0}$ runs into great technical difficulty: it is impossible to find eigenvalues of $\tilde{\mu}, \tilde{M}$, and $u_{1}$ to the necessary accuracy.

The positions of minima of the energy (28) on the plane $\left\{f_{2}, \tilde{E}\right\}$ are illustrated by Fig. 9 for different values of $\tilde{\xi}_{0}$. Notice that numerical errors in calculating magnitudes and positions of minima of the energy $\Delta\left(\tilde{\xi}_{0}\right)$ are quite large, and perhaps this leads to shifting the points with $\Delta\left(\tilde{\xi}_{0}=0.6,0.8\right)$ and $\Delta\left(\tilde{\xi}_{0}=1.1,1.5\right)$.

Searching for the mass gap $\Delta\left(\tilde{\xi}_{0}\right)$ for $\tilde{\xi}_{0}>4.5$ runs into the aforementioned technical problems. For a qualitative understanding what can happen for large $\tilde{\xi}_{0}$, we have investigated dependencies of the total energy, $\tilde{W}_{t}$, and of the energies of the monopole, $\left(\tilde{W}_{t}\right)_{\mathrm{Pm}}$, and of the spinball, $\left(\tilde{W}_{t}\right)_{s}$, on the parameter $\tilde{\xi}_{0}$ for fixed values of the parameters $f_{2}$ and $\tilde{E}$. Taking into account the formulas (16) and (18), the quantities $\left(\tilde{W}_{t}\right)_{s}$ and $\left(\tilde{W}_{t}\right)_{\mathrm{Pm}}$ describe the total energy of the system under investigation as a sum of the energies of the Proca monopole and of the spinball: $\tilde{W}_{t}=\left(\tilde{W}_{t}\right)_{\mathrm{Pm}}+\left(\tilde{W}_{t}\right)_{s}$. The results of calculations are given in Fig. 11. It is seen that there are local minima for all the energies for some values of $\tilde{\xi}_{0}$. The behavior of these quantities when the parameter $\tilde{\xi}_{0}$ increases further is unclear, and it requires further investigations. From an analysis of the graphs, we can assume that $\left(\tilde{W}_{t}\right)_{s}$ will tend to zero and $\left(\tilde{W}_{t}\right)_{\mathrm{Pm}}$ will tend to a constant value as $\tilde{\xi}_{0} \rightarrow \infty$; this would mean that $\tilde{W}_{t} \stackrel{\tilde{\xi}_{0} \rightarrow \infty}{\rightarrow}$ const for fixed $f_{2}$ and $\tilde{E}$. This observation permits us to assume that such a behavior persists for all values of $f_{2}$ and $\tilde{E}$; this may result in the existence of a mass gap in the energy spectrum of the particlelike solutions under investigation within a classical field theory containing a non-Abelian Proca field plus nonlinear Higgs and Dirac fields.

For understanding the behavior of the energies $\tilde{W}_{t}$, $\left(\tilde{W}_{t}\right)_{\mathrm{Pm}}$, and $\left(\tilde{W}_{t}\right)_{s}$ as functions of $\tilde{\xi}_{0}$, we show in Fig. 12 the graphs of the functions $f(x), \tilde{\xi}(x), \tilde{u}(x) / x$, and $\tilde{v}(x) / x$. It is seen that with increasing $\tilde{\xi}_{0}$ characteristic magnitudes of the functions $f$ and $\tilde{\xi}$ decrease, while those of the functions $\tilde{u} / x$ and $\tilde{v} / x$ increase. Such a behavior of the functions leads at least to not decreasing (and maybe even to increasing) the energy of the Proca monopole, which basically depends on the functions $f$ and $\tilde{\xi}$, and to increasing the energy of the spinball, which basically depends on the functions $\tilde{v}$ and $\tilde{u}$. For large values of $\tilde{\xi}_{0}$, the solutions are derived by matching numerical solutions (which are obtained up to some radius $x_{1}$ ) with the corresponding asymptotic functions from (23) and (25).

\section{DISCUSSION AND CONCLUSIONS}

We have considered non-Abelian SU(2) Proca-DiracHiggs theory where a massive vector field interacts with nonlinear scalar and spinor fields. Within such theory, regular, nontrivial particlelike solutions describing finite-energy configurations-Proca-monopole-plusspinball systems-have been constructed. A distinctive feature of the Proca monopole is that, unlike the 't Hooft-Polyakov monopole, the corresponding color field decays exponentially at infinity. In our opinion, the main reason why such solutions can exist is the presence of the mass of the non-Abelian Proca field, and also because of the structure of the Dirac equation leading to the existence of regular solutions of this equation.

Note that both a Proca monopole and a spinball can exist separately; i.e., one can get a Proca monopole as a solution of Eqs. (10) and (11) with $\tilde{u}=\tilde{v}=0$. Also, there exists a particlelike solution (a spinball) of the nonlinear Dirac equation (12) and (13) with $f=1$ and $\xi=$ const (see Refs. [11,12]). The basic distinction between these two solutions is that the energy spectrum of the Proca monopole does not have a mass gap, while for the particlelike solution of the nonlinear Dirac equation such a gap does exist $[11,12]$. This means that if the mass gap exists for the complete set of equations (10)-(13), this is a consequence of the presence of the nonlinear Dirac field. Note in this connection that in the 1950s the mass gap has in fact been found in Refs. [11,12] in solving the nonlinear Dirac equation. However, the authors did not use such a term, but said of "the lightest stable particle". Those papers were devoted to study of the nonlinear Dirac equation, and W. Heisenberg offered to use it as a fundamental equation in describing the properties of an electron. To the best of our knowledge, the mass gap was first obtained in Refs. [11,12].

We have calculated the energy spectrum of the corresponding solutions for fixed values of $\tilde{\xi}_{0}$ and have shown that there is a minimum value of the energy [the mass gap $\Delta\left(\tilde{\xi}_{0}\right)$ for a fixed $\left.\tilde{\xi}_{0}\right]$. Also, we have studied the dependence of $\Delta$ on $\tilde{\xi}_{0}$ in the range $0.4<\tilde{\xi}_{0}<4.5$. In order to understand whether the mass gap exists in a whole range of possible values of $\tilde{\xi}_{0}$, we have examined the behavior of the total energy of the particlelike solution, as well as the behavior of the energies of the Proca monopole and of the spinball, which make up the particlelike configuration under consideration, for some fixed values of the parameters $f_{2}$ and $\tilde{E}$. As a result, we have shown that these energies have local minima, and the energy of the Proca monopole possibly tends to a constant value. This permits us to suggest that such a behavior will occur for any values of $f_{2}$ and $\tilde{E}$; this eventually will lead to the appearance of a mass gap in a whole range of values of the parameters $f_{2}$, $\tilde{E}$, and $\tilde{\xi}_{0}$ determining the characteristics of the particlelike solutions under investigation.

Thus, one of the purposes of this paper is to study the energy spectrum of particlelike solutions and to obtain the mass gap $\Delta\left(\xi_{0}\right)$ for a fixed $\xi_{0}$. All this permits us to understand the mechanism of the occurrence of a mass gap in Proca-Dirac-Higgs theory: the reason is that the Dirac equation is nonlinear. Bearing this in mind, one can assume 
that a similar mechanism may also be responsible for the appearance of a mass gap in QCD. For this, however, one has to understand how the nonlinear Dirac equation may occur in QCD. This can happen as follows. In the Lagrangian, the interaction between quarks and gluons is described by the term $\hat{\bar{\psi}} \lambda^{B} \hat{A}_{\mu} \hat{\psi}$, where $\hat{\psi}=\langle\hat{\psi}\rangle+\widehat{\delta \psi}$, $\hat{A}_{\mu}=\left\langle\hat{A}_{\mu}\right\rangle+\widehat{\delta A}_{\mu},\langle\hat{\psi}\rangle,\left\langle\hat{A}_{\mu}\right\rangle$ are valence quarks and gluons, and $\widehat{\delta \psi}, \widehat{\delta A}_{\mu}$ are sea quarks and gluons; $\lambda^{B}$ is the Gell-Mann matrices. One can assume that the quantum average of the term $\left\langle\widehat{\delta \bar{\psi}} \lambda^{B} \widehat{\delta A}_{\mu} \widehat{\delta \psi}\right\rangle$ will approximately look like $\left\langle\widehat{\delta \bar{\psi}} \lambda^{B} \widehat{\delta A}_{\mu} \widehat{\delta \psi}\right\rangle \approx \phi(\bar{\xi} \xi)^{2}$, where the field $\phi$ approximately describes the sea gluons and the spinor field $\xi$ approximately describes the sea quarks. Thus, in QCD, there can occur the nonlinear Dirac equation which approximately describes the interaction between sea quarks and gluons. Summarizing, one can say that the following mechanism of the occurrence of a mass gap in QCD is suggested: the nonperturbative interaction between sea quarks and gluons is approximately described by a nonlinear spinor field, the presence of which leads in turn to the occurrence of a mass gap in QCD.

Thus, the following results have been obtained:

(i) Particlelike solutions of the type Proca-monopoleplus-spinball have been found in some range of values of the system parameters $f_{2}, \tilde{E}$, and $\tilde{\xi}_{0}$.

(ii) For such solutions, the energy spectra for some values of the parameter $\tilde{\xi}_{0}$ have been constructed. It was shown that they possess a minimum $\Delta\left(\tilde{\xi}_{0}\right)$.

(iii) The behavior of $\Delta\left(\tilde{\xi}_{0}\right)$ as a function of the parameter $\tilde{\xi}_{0}$ has been studied.

(iv) It was shown that the solutions obtained give rise to a Meissner-like effect, which consists in the fact that a maximum value of the SU(2) gauge Proca field is located there where the Higgs field has a minimum.

(v) For the Proca monopole, it was shown that the Proca color "magnetic" field decreases asymptotically according to an exponential law.

(vi) The non-Abelian Proca monopole obtained differs in principle from the 't Hooft-Polyakov monopole in the sense that the Proca monopole is topologically trivial.

(vii) The main reason for the existence of the mass gap $\Delta\left(\tilde{\xi}_{0}\right)$ is due to the presence of the nonlinear Dirac field.

(viii) The mechanism of the occurrence of a mass gap in QCD has been suggested.

Note that when one tries to prove the presence (or absence) of the mass gap $\Delta$, he/she encounters great technical difficulties related to the fact that with increasing $\tilde{\xi}_{0}$ the mass gap $\Delta\left(\tilde{\xi}_{0}\right)$ shifts towards $f_{2} \rightarrow 0$ and $\tilde{E} \rightarrow m_{f}$.
But for such values of $f_{2}$ and $\tilde{E}$ the eigenvalues $\tilde{\mu}, \tilde{M}$, and $\tilde{u}_{1}$ should be given to high accuracy, leading to such technical problems. In order to assume what can happen with the function $\Delta\left(\tilde{\xi}_{0}\right)$ with increasing $\tilde{\xi}_{0}$, we have studied the behavior of the total energy $\tilde{W}_{t}$, as well as the energies of the Proca monopole, $\left(\tilde{W}_{t}\right)_{\mathrm{Pm}}$, and of the spinball, $\left(\tilde{W}_{t}\right)_{s}$, as functions of $\tilde{\xi}_{0}$ for some fixed values of the parameters $f_{2}, \tilde{E}$ and have shown that in this case all the energies have a minimum (at least a local one). This allows us to hope that such a minimum will occur for any values of the parameters $f_{2}$ and $\tilde{E}$; this in turn assumes that there will be a minimum value of the energy $\tilde{W}_{t}$ in a whole range of values of the parameters $f_{2}, \tilde{E}$, and $\tilde{\xi}_{0}$.

Numerical study of the energy spectrum of a spinball [when one solves only Eqs. (12) and (13)] indicates that its energy goes to infinity when $\tilde{E} \rightarrow \tilde{m}_{f}$ and when $\tilde{E} \rightarrow 0$; this corresponds to the fact that somewhere inside this region there is a minimum value of the energy (a mass gap of the spinball). Our numerical calculations indicate that apparently when Proca and Higgs fields are applied the energy of our system also goes to infinity, but now when $\tilde{E} \rightarrow \tilde{m}_{f}$ or when $f_{2}$ tends to some critical value $f_{2}=\left(f_{2}\right)_{\mathrm{cr}}$.

Taking into account all the above, the existence of a mass gap within a theory containing a SU(2) Proca field plus nonlinear Higgs and Dirac fields is not impossible. To confirm this, it is necessary to carry out further investigations in this direction which are connected with large technical problems associated with solving a nonlinear eigenvalue problem when eigenvalues must be determined to high accuracy.

Finally, note that the particlelike solutions obtained here can be considered as those describing a spinorial Proca glueball. In QCD, a glueball is a hypothetical particle consisting only of a gauge Yang-Mills field in the absence of quarks. In our case a spinor field is present, but this is a nonlinear field that does not describe quarks. Since the solutions obtained are supported by non-Abelian Proca and nonlinear Higgs and Dirac fields, we can refer to a configuration described by such solutions as a spinorial Proca glueball (or a Proca-monopole-plus-spinball system).

\section{ACKNOWLEDGMENTS}

This work was supported by Grant No. BR05236730 in Fundamental Research in Natural Sciences by the Ministry of Education and Science of the Republic of Kazakhstan. V.D. and V.F. also are grateful to the Research Group Linkage Programme of the Alexander von Humboldt Foundation for the support of this research. 
[1] R. Scipioni, Classical Quantum Gravity 16, 2471 (1999).

[2] R. Tomaschitz, Eur. Phys. J. D 32, 241 (2005).

[3] S. Ponglertsakul and E. Winstanley, Phys. Rev. D 94, 044048 (2016).

[4] R. Brito, V. Cardoso, C. A. R. Herdeiro, and E. Radu, Phys. Lett. B 752, 291 (2016).

[5] C. A. R. Herdeiro, A. M. Pombo, and E. Radu, Phys. Lett. B 773, 654 (2017).

[6] L. Heisenberg, J. Cosmol. Astropart. Phys. 05 (2014) 015.

[7] E. Allys, P. Peter, and Y. Rodriguez, J. Cosmol. Astropart. Phys. 02 (2016) 004.

[8] A. J. Silenko, Phys. Rev. D 98, 025014 (2018).
[9] A. de Felice, L. Heisenberg, and S. Tsujikawa, Phys. Rev. D 95, 123540 (2017).

[10] E. Allys, P. Peter, and Y. Rodriguez, Phys. Rev. D 94, 084041 (2016).

[11] R. Finkelstein, R. LeLevier, and M. Ruderman, Phys. Rev. 83, 326 (1951).

[12] R. Finkelstein, C. Fronsdal, and P. Kaus, Phys. Rev. 103, 1571 (1956).

[13] X. z. Li, K. 1. Wang, and J. z. Zhang, Nuovo Cimento A 75, 87 (1983).

[14] K.1. Wang and J.z. Zhang, Nuovo Cimento A 86, 32 (1985). 